\title{
Flow and Noise Control in High Speed and High Reynolds Number Jets Using Plasma Actuators
}

M. Samimy, J. Kastner, J.-H. Kim, Y. Utkin, and I. Adamovich

The Ohio State University, Columbus, Ohio

C.A. Brown

Glenn Research Center, Cleveland, Ohio 


\section{NASA STI Program . . . in Profile}

Since its founding, NASA has been dedicated to the advancement of aeronautics and space science. The NASA Scientific and Technical Information (STI) program plays a key part in helping NASA maintain this important role.

The NASA STI Program operates under the auspices of the Agency Chief Information Officer. It collects, organizes, provides for archiving, and disseminates NASA's STI. The NASA STI program provides access to the NASA Aeronautics and Space Database and its public interface, the NASA Technical Reports Server, thus providing one of the largest collections of aeronautical and space science STI in the world. Results are published in both non-NASA channels and by NASA in the NASA STI Report Series, which includes the following report types:

- TECHNICAL PUBLICATION. Reports of completed research or a major significant phase of research that present the results of NASA programs and include extensive data or theoretical analysis. Includes compilations of significant scientific and technical data and information deemed to be of continuing reference value. NASA counterpart of peer-reviewed formal professional papers but has less stringent limitations on manuscript length and extent of graphic presentations.

- TECHNICAL MEMORANDUM. Scientific and technical findings that are preliminary or of specialized interest, e.g., quick release reports, working papers, and bibliographies that contain minimal annotation. Does not contain extensive analysis.

- CONTRACTOR REPORT. Scientific and technical findings by NASA-sponsored contractors and grantees.
- CONFERENCE PUBLICATION. Collected papers from scientific and technical conferences, symposia, seminars, or other meetings sponsored or cosponsored by NASA.

- SPECIAL PUBLICATION. Scientific, technical, or historical information from NASA programs, projects, and missions, often concerned with subjects having substantial public interest.

- TECHNICAL TRANSLATION. Englishlanguage translations of foreign scientific and technical material pertinent to NASA's mission.

Specialized services also include creating custom thesauri, building customized databases, organizing and publishing research results.

For more information about the NASA STI program, see the following:

- Access the NASA STI program home page at http://www.sti.nasa.gov

- E-mail your question via the Internet to help@sti.nasa.gov

- Fax your question to the NASA STI Help Desk at 301-621-0134

- Telephone the NASA STI Help Desk at 301-621-0390

- Write to:

NASA STI Help Desk

NASA Center for AeroSpace Information 7115 Standard Drive Hanover, MD 21076-1320 


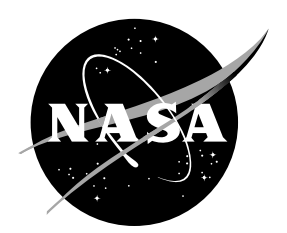

\title{
Flow and Noise Control in High Speed and High Reynolds Number Jets Using Plasma Actuators
}

\author{
M. Samimy, J. Kastner, J.-H. Kim, Y. Utkin, and I. Adamovich
}

The Ohio State University, Columbus, Ohio

C.A. Brown

Glenn Research Center, Cleveland, Ohio

Prepared for the

Third Flow Control Conference

sponsored by the American Institute of Aeronautics and Astronautics

San Francisco, California, June 5-8, 2006

National Aeronautics and

Space Administration

Glenn Research Center

Cleveland, Ohio 44135 


\section{Acknowledgments}

The support of the research at The Ohio State University by the NASA Glenn Research Center and the Ohio Department of Development is greatly appreciated. We greatly appreciate the help from James Bridges in many facets of this research.

Trade names and trademarks are used in this report for identification only. Their usage does not constitute an official endorsement, either expressed or implied, by the National Aeronautics and Space Administration.

This work was sponsored by the Fundamental Aeronautics Program at the NASA Glenn Research Center.

Level of Review: This material has been technically reviewed by technical management.

Available from

NASA Center for Aerospace Information 7115 Standard Drive

Hanover, MD 21076-1320
National Technical Information Service 5285 Port Royal Road Springfield, VA 22161 


\title{
Flow and Noise Control in High Speed and High Reynolds Number Jets Using Plasma Actuators
}

\author{
M. Samimy, J. Kastner, J.-H. Kim, Y. Utkin, and I. Adamovich \\ The Ohio State University \\ Columbus, Ohio 43235 \\ C.A. Brown \\ National Aeronautics and Space Administration \\ Glenn Research Center \\ Cleveland, Ohio 44135
}

\begin{abstract}
The idea of manipulating flow to change its characteristics is over a century old. Manipulating instabilities of a jet to increase its mixing and to reduce its radiated noise started in the 1970s. While the effort has been successful in low-speed and low Reynolds number jets, available actuators' capabilities in terms of their amplitude, bandwidth, and phasing have fallen short in control of high-speed and high Reynolds number jets of practical interest. Localized arc filament plasma actuators have recently been developed and extensively used at Gas Dynamics and Turbulence Laboratory (GDTL) for control of highspeed and high Reynolds number jets. While the technique has been quite successful and is very promising, all the work up to this point had been carried out using small high subsonic and low supersonic jets from a $2.54 \mathrm{~cm}$ diameter nozzle exit with a Reynolds number of about a million. The preliminary work reported in this paper is a first attempt to evaluate the scalability of the technique. The power supply/plasma generator was designed and built in-house at GDTL to operate 8 actuators simultaneously over a large frequency range ( 0 to $200 \mathrm{kHz})$ with independent control over phase and duty cycle of each actuator. This allowed forcing the small jet at GDTL with azimuthal modes $m=0,1,2,3$, $\pm 1, \pm 2$, and \pm 4 over a large range of frequencies. This power supply was taken to and used, with minor modifications, at the NASA Nozzle Acoustic Test Rig (NATR). At NATR, 32 actuators were distributed around the $7.5 \mathrm{in}$. nozzle (a linear increase with nozzle exit diameter would require 60 actuators). With this arrangement only 8 actuators could operate simultaneously, thus limiting the forcing of the jet at NATR to only three azimuthal modes $\mathrm{m}= \pm 1,4$, and 8 . Very preliminary results at NATR indicate that the trends observed in the larger NASA facility in terms of the effects of actuation frequency and azimuthal modes are similar in both small GDTL and larger NASA jets. However, the actuation authority seems to fall short in the larger jet at higher Mach numbers, resulting in decreased amplitude response compared to the small jet, which is attributed at this point to the lack of sufficient number of actuators. The preliminary results seem also to suggest that amplitude of actuation tones is similar in both the small and larger jets.
\end{abstract}

\section{Introduction}

\section{Excitation of Jets for Aeroacoustics}

The idea of manipulating the turbulence of jets for engineering benefit has been around for many decades. The promises for reducing jet noise, increasing mixing efficiency, and other applications have lead many to pursue actuator technologies which would bring these promises to fruition. Along the way, many problems have been found, often associated with the actuators being used. 


\section{The Promise}

For jet noise, control of jet turbulence has had two potential benefits: enhanced understanding of noise generation mechanisms and direct manipulation of these mechanisms for noise reduction. It is widely observed that jet noise scales as a high power of jet velocity $U_{j}$. The acknowledged $U_{j}^{8}$ law can be viewed as a conversion law for transforming energy from turbulence to acoustics (Lighthill 1952, 1954).

The coefficient in front of this exponential factor is also known to be very small, somewhere around $10^{-7}$. The happy fact that most turbulent kinetic energy does not convert to sound is a result of the conservation laws for angular momentum, which at low Mach numbers $(M \sim 0)$ has each noise-producing impulse countered by another, oppositely directed impulse. This cancellation becomes less complete as Mach number increases, but still remains nearly perfect as one compares the energy available with that radiated. It also explains why the study of jet noise remains active today as researchers strive to understand and model this one in a few million portion of the flow which does radiate sound. It has also been shown theoretically that, for small Mach numbers, high order azimuthal undulations of the jet shear layer do not couple effectively with the acoustic field, making them particularly inefficient noise generators (Michalke and Fuchs 1975). The other aspect of jet noise research that has precluded understanding is that the turbulence has only been studied in statistical fashion, the motions which produce the noise being nondeterministic.

To date, almost all jet noise reduction has been accomplished by reducing the jet exit velocity. This has been done by increasing bypass ratios of the engines, through improvements in materials and engine core architecture. But what if the efficiency of the conversion of turbulence to noise, while admittedly small, could be reduced even further? One possibility is to remove the statistically rare, but acoustically significant events that have been found to produce most of the noise in the jet. Perhaps if the turbulent structures could be controlled to further maximize the cancellation between impulse-carrying structures, the efficiency would be reduced even further. Another possibility is to pump more of the jet's turbulent kinetic energy into high-order azimuthal modes, putting more of the kinetic energy into dynamic motions that are inefficient at making noise. Both of these possibilities require actuators with control authority greater than that imposed by the non-deterministic turbulence found in the jet.

Even if ultimately we cannot find a way to reduce noise by controlling flow structures in a jet, it is still of interest to study how these structures relate to the generation of sound in jets. The promise of jet excitation for jet noise theory is that if the researcher could somehow remove the non-deterministic portion of the problem, then the mechanisms of noise production could be studied directly and with much greater precision. This is a much more realistic application of jet control technology, as requiring control of laboratory jets is somewhat more reasonable than implementing control on jet aircraft with their multiple constraints.

\section{The Problems}

The insight of Brown and Roshko (1974) that shear layer turbulence is composed of large, wellorganized structures even in high Reynolds numbers is usually credited with starting the study of largescale, 'coherent' structures and their control. During the 1970's and 1980's many studies were done where free shear flows, particularly jets, were perturbed using actuators, and their response studied (Crow and Champagne 1971, Moore 1977, Kibens 1980, Zaman and Hussain 1981, Ho and Huerre 1984, Ahuja and Blakney 1985). Most of these studies were done in low Reynolds number jets using acoustic drivers as actuators. However, it was found that the acoustic driver actuators did not retain control authority as either the jet diameter or speed (thus Reynolds number) increased. Jet noise could not be readily studied because scale limitations did not allow high enough Mach number flows without Reynolds numbers above where the acoustic drivers lost effectiveness. In short, the actuators did not scale up adequately to be useful in either research or reduction concepts. 


\section{Actuator Coupling}

To discuss further the difficulties faced by any actuator to be used on a jet and the motivation for the current work, details of how actuators couple with the flow to manipulate it must be discussed. The way to control the turbulent structures in a jet is through the flow instabilities which govern their formation and interactions. Two dominant instabilities have been identified to date, the initial shear layer instability and jet column instability.

The initial shear layer instability.- The first type of instability is of an intrinsically two-dimensional shear layer and is applicable in regions of the jet near the nozzle where curvature is negligible compared with the shear layer momentum thickness the main scaling dimension. Although there is some sensitivity to the shape factor of the velocity profile, the maximum amplification of the instability has a Strouhal number, $f_{0} \theta_{0} / U_{j} \approx 0.013$. This often corresponds to the first formation of vortices in the jet, which gives rise to subsequent vortex interactions. The decimation of vortices by interactions, while not as clean a process as simple vortex pairing, does result in spectral peaks which continue to scale with momentum thickness as the jet shear layer evolves down the potential core. Excitation of the shear layer mode has been successful in the past either by modulation of the mean jet flow or more efficiently by deflection of the initial separation point on the nozzle. Given that many small laboratory jets have momentum thickness that scales as $D R e^{-1 / 2}, S t_{\theta} \approx 0.013$ corresponds to $40 \mathrm{kHz}$ for a $1 \mathrm{in}$. jet at Mach 0.5 .

Jet column instability.- The second type of instability is that of the jet column, where the applicable scaling dimension is the diameter of the column. This instability scaling is clearly found at and beyond the end of the jet potential core and accounts for the dominant spectral peak near Strouhal number $f D / U_{J} \approx 0.3$ found in this region. This instability supports several azimuthal modes, which after the first few become successively weaker in their amplification. The jet column instability has been successfully controlled in the past by directly modulating the axial flow in the potential core using acoustic methods.

Perhaps it is obvious that the shear layer modes and jet column modes must compete near the end of the potential core where their regions of influence intersect. This interaction can be quite dramatic if they occur such that the shear layer mode has the right shape and frequency to directly reinforce the jet column mode. This is often the case in small, low speed jet facilities where much of the previous work on excited jets has taken place. It is less likely to occur in large jets, and partly explains why actuators for jets have not been as successful at higher Reynolds numbers.

Energy and size considerations.-Because the shear layer instability scales with local momentum thickness, the scale-up of this type of excitation should be straightforward. Techniques that perturb the flow by oscillation of the initial shear layer separation need only be scaled by the perimeter of the nozzle and the exit boundary layer thickness of the nozzle. If we assume that the initial boundary layer thickness scales by the square root of scale factor and the perimeter by the scale factor, then the energy required goes up with size as scale factor raised to the power 1.5. Increasing the number of actuators to keep the energy density on the nozzle lip constant nearly accounts for the required scale-up of energy, with the rest perhaps accounted for by an increase in actuator strength, depending upon the actual boundary layer properties. Likewise, the frequencies required will be determined by the momentum thickness, not the scale factor directly.

The jet column, being primarily controlled by global modes, is much harder to excite as the jet becomes larger. Here the impact of the actuator on the jet must not only scale with the perimeter, but perhaps with the area of the cross-section. To effect a modulation of the potential core requires energy scaling perhaps with the jet area, not the diameter. It is also much less likely that shear layer instability modes can couple with the jet column modes as they scale on boundary layer thickness, not nozzle diameter.

Another consideration in applying control actuators to jets for noise reduction purposes is the noise of the actuators themselves. Many potential actuators produce more noise than they remove at laboratory scale. However, if one scales up the actuators linearly with the perimeter of the jet, then the actuator selfnoise increases linearly while the noise of the jet and the noise removed scales with area of the jet. Depending upon how the actuators scale up, actuator self-noise may or may not be a problem. 


\section{Motivation of Present Work}

To summarize, it seems apparent from simple scaling arguments that, for instability excitation techniques to be applicable to jets of practical size, energetic actuators are critical. Thus, while previous application of the current technique, plasma arc excitation, has proven useful for research into jet dynamics in small scale laboratory environment, it is important to understand whether this technique can be brought to larger scale research facilities for higher fidelity tests, and eventually to full-scale engine applications.

The presentation of this paper will focus on application of the plasma arc jet excitation to the classically understood jet instabilities, first in the small-scale jet rig at the Gas Dynamics and Turbulence Laboratory (GDTL) at The Ohio State University (OSU), and then where possible in the much bigger jet rig at NASA Glenn Research Center. The objectives are to demonstrate that the actuator concept works in the small-scale rig, delivering results at much greater Reynolds numbers than have been possible with previous techniques, and to demonstrate the degree to which the actuators work when taken to a much larger scale jet. Certain limitations, not the least of which was test time, precluded extensive comparisons in the big facility, but the initial results are encouraging that the plasma arc jet excitation technique may be able to control jets of larger Reynolds numbers, including that of full-scale aircraft.

The major limitation was the number of actuators that could be operated simultaneously. The power supply/plasma generator was designed and built in-house at GDTL to operate 8 actuators simultaneously over a large frequency range (0 to $200 \mathrm{kHz}$ ) with independent control over phase and duty cycle of each actuator (Samimy et al. 2006a and b). This allowed forcing the small 1 in. diameter jet at GDTL with azimuthal modes $m=0,1,2,3, \pm 1, \pm 2$, and \pm 4 . A decision was made to take this power supply to NASA and distribute 32 actuators around the $7.5 \mathrm{in}$. jet at NASA (a linear increase would require 60 actuators). With this arrangement only 8 actuators could operate simultaneously, thus limiting the forcing of the jet at NASA to only three azimuthal modes $m= \pm 1,4$, and 8 . Obviously this arrangement along with relatively short period of time allowed for the experiment imposed significant limitation on what could be achieved. The results presented below must be viewed with these limitations in mind.

\section{Nomenclature}

$c_{j} \quad$ Speed of sound in jet

$c_{o} \quad$ Ambient speed of sound

$D \quad$ Nozzle exit diameter

$F \quad$ Frequency

$f_{0} \quad$ Most amplified initial shear layer frequency

$f_{F} \quad$ Forcing (excitation) frequency

$f_{p} \quad$ Jet preferred mode frequency

$m \quad$ Azimuthal mode number

$M \quad$ Gas dynamics Mach number, $U_{j} / c_{j}$

$M_{a} \quad$ Acoustic Mach number, $U_{j} / c_{o}$

$R e_{D} \quad$ Reynolds number, $U_{j} D / v$

$S t_{D} \quad$ Strouhal number based on the nozzle exit diameter, $f D / U_{j}$

$S t_{D F} \quad$ Strouhal number based on the excitation frequency, $f_{F} D / U_{j}$

$S t_{\theta} \quad$ Strouhal number based on boundary layer momentum thickness at the nozzle exit, $f_{0} \theta_{0} / U_{j}$

$U_{j} \quad$ Nozzle exit velocity (jet centerline velocity within the potential core)

$x \quad$ Distance from the nozzle exit along the jet centerline (fig. 1)

$y \quad$ Vertical distance from the jet centerline (fig. 1)

$\theta \quad$ Angle with respect to downstream jet axis (x-axis)

$\theta_{0} \quad$ Momentum thickness at the nozzle exit or at the trailing edge of the splitter plate 

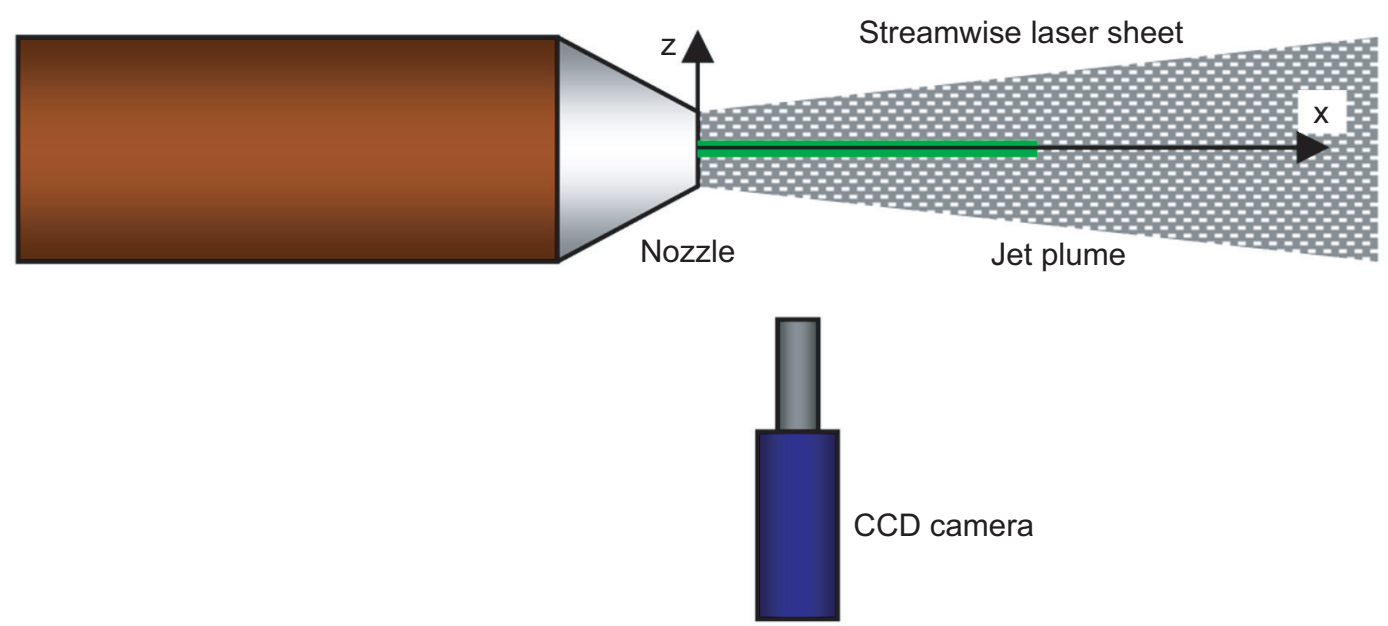

Figure 1.-Schematic of the jet and the PIV set up at GDTL. Y-coordinate is normal to the plane.

\section{Experimental Facility and Techniques}

\section{Flow Facilities}

Two sets of experiments were conducted: one set in the smaller facility of GDTL to obtain detailed results on the application of plasma actuators for flow and acoustic control and the second set in the larger NASA Glenn Research Center facility to explore the scalability of the technique. A brief description of each facility will be given here. More detailed descriptions of GDTL and NASA facilities can be found in Samimy et al. (2006b) and in Bridges and Wernet (2004). Also, additional and more detailed results on flow and noise control using plasma actuators at GDTL can be found in Samimy et al. (2006a and b) and Kastner et al. (2006).

At GDTL, the ambient air is compressed, dried, and stored in two cylindrical tanks at a pressure of up to $16 \mathrm{MPa}$ with a capacity of $36 \mathrm{~m}^{3}$. The compressed air is supplied to the stagnation chamber of the jet facility and conditioned before entering into a nozzle. Either a converging or a converging-diverging nozzle could be used and the jet could be operated continuously from low subsonic to Mach 2.5. An axisymmetric converging nozzle was used for the experiments discussed in this paper and the acoustic Mach number was varied in the high subsonic range ( $M_{a}$ from 0.5 to 0.84$)$. The air is discharged horizontally through the nozzle into an anechoic chamber (fig. 1). The exit diameter of the nozzle is $2.54 \mathrm{~cm}$ (1.0 in.). A nozzle extension, made of boron nitride, was attached to the exit of the nozzle to house the plasma actuators.

Experiments were conducted on the dual flow jet rig in the Nozzle Acoustic Test Rig (NATR) at the NASA Glenn Research Center's Aeroacoustic Propulsion Laboratory. This dual flow engine simulator provides exhaust gases at pressure and temperature conditions typical of modern turbofan engines with mass flows up to $65 \mathrm{~kg} / \mathrm{sec}$. The NATR is a $1.35 \mathrm{~m}$ free jet in which the jet rig sits, providing a moving free stream around the jet plume at speeds up to $M=0.35$. For this set of experiments, a nozzle system typical of internally mixed engine exhaust systems with a confluent axisymmetric splitter was used and both streams were run at the same pressure ratio without heating the air. Also, no forward flight stream was employed, creating a basic single-stream static jet to compare with the GDTL facility. The final section of the nozzle was replaced with a boron nitride piece having the same internal flow lines as the high-fidelity nozzle, but with a much fatter lip to house the plasma actuator electrodes in a fashion similar to the GDTL hardware. A photograph of the plasma actuators mounted on at the NASA Nozzle Acoustic Test Rig is shown in figure 2. 


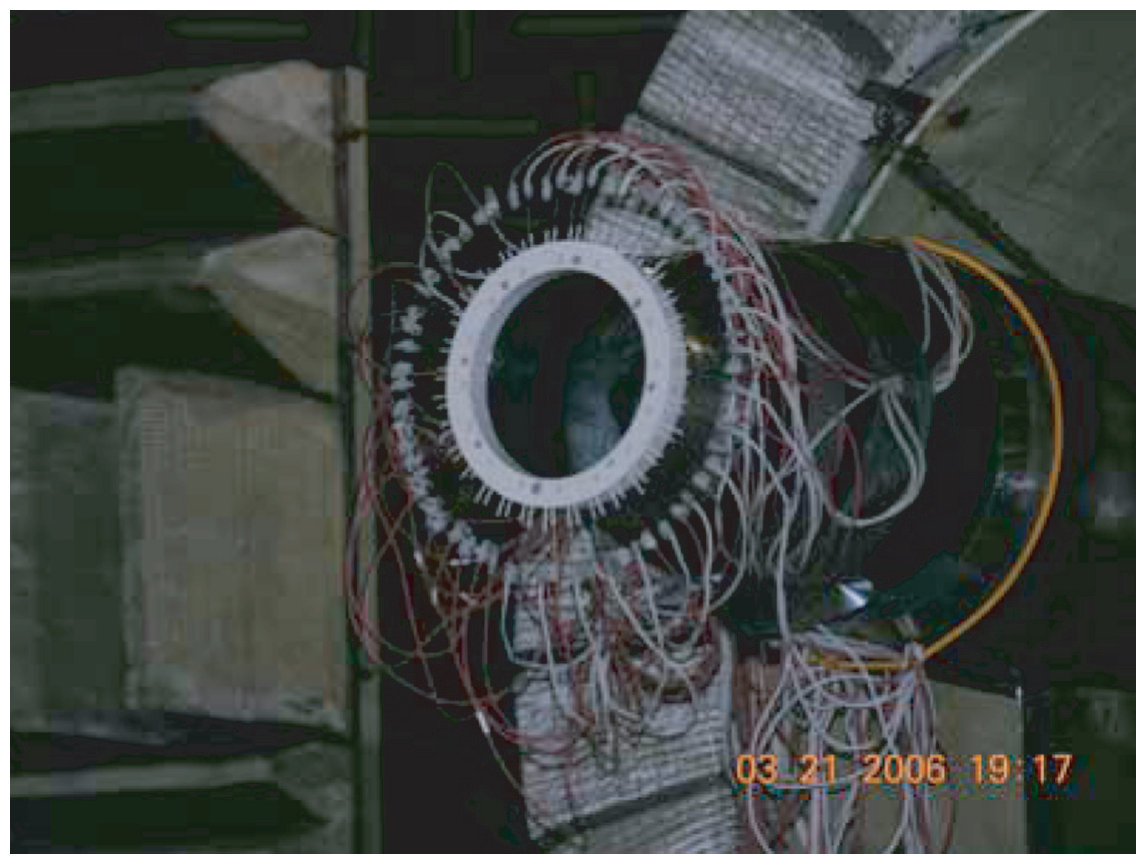

Figure 2.-Photograph of the plasma actuators mounted on at the NASA Nozzle Acoustic Test Rig.

TABLE I.-NOZZLE EXIT DIAMETER AND JET FLOW VARIABLES FOR GDTL AND NASA FACILITIES

\begin{tabular}{|c|c|c|c|c|c|c|c|}
\hline & \multicolumn{7}{|c|}{ VARIABLES FOR GDTL AND NASA FACILITIES } \\
\hline & $\begin{array}{l}D, \\
\mathrm{~cm}\end{array}$ & $M_{a}$ & $M$ & $R e_{D}$ & $\begin{array}{c}U_{j}, \\
\mathrm{~m} / \mathrm{s}\end{array}$ & $\begin{array}{c}f_{F}, \\
\mathrm{kHz} \\
\left(S t_{D F}=0.3\right)\end{array}$ & $\begin{array}{c}f_{F}, \\
\mathrm{kHz} \\
\left(S t_{D F}=1.8\right)\end{array}$ \\
\hline \multirow[t]{3}{*}{ GDTL } & 2.54 & 0.5 & 0.51 & $3.8 \times 10^{5}$ & 166 & 2.0 & 11.8 \\
\hline & & .7 & .74 & $5.7 \times 10^{5}$ & 235 & 2.8 & 16.6 \\
\hline & & .84 & .9 & $7.2 \times 10^{5}$ & 285 & 3.4 & 20.2 \\
\hline \multirow[t]{4}{*}{ NASA } & 19.05 & .5 & .51 & $2.9 \times 10^{6}$ & 166 & 0.3 & 1.6 \\
\hline & & .7 & .74 & $4.3 \times 10^{6}$ & 235 & .4 & 2.2 \\
\hline & & .84 & .9 & $5.5 \times 10^{6}$ & 285 & .4 & 2.7 \\
\hline & & .9 & .99 & $6.4 \times 10^{6}$ & 301 & .5 & 2.8 \\
\hline
\end{tabular}

Table I depicts the nozzle exit diameter, and the jet acoustic and gas dynamic Mach numbers, velocity, and Reynolds number for the jets at GDTL and NASA used for the work presented in this paper. The boundary layer thickness at the exit of the nozzle at GDTL is very thin, making it almost impossible to obtain a boundary layer profile to determine its momentum thickness and its state. Kastner et al. (2004) used a similar converging nozzle and measured velocity at a few points within the boundary layer of an $M=0.9$ jet. They estimated the boundary layer to be turbulent with a thickness of about $1 \mathrm{~mm}$ and a momentum thickness of approximately $0.1 \mathrm{~mm}$. The characteristics of the boundary layer in the current experiments are expected to be quite similar at similar Mach numbers. At the lower Mach numbers, especially at $M_{a}=0.5$, the boundary is probably laminar. Also shown in the table are the forcing frequencies at GDTL and NASA, which were adjusted to keep the same forcing Strouhal number $\left(S t_{D F}\right)$. For example, for the $M_{a}=0.5$ jet, the forcing frequency for forcing Strouhal number $S t_{D F}=0.3 \mathrm{was} 2 \mathrm{kHz}$ at GDTL and $300 \mathrm{~Hz}$ at NASA. 
An important point about scale up on actuator concepts becomes clear when considering the last column of Table I. Taking the same actuator design (and indeed actual hardware) to a much larger jet requires great flexibility as the frequencies change dramatically when Strouhal numbers are kept constant. The boundary layer of the NASA rig was not measured, but is sure to be fully turbulent given the relatively small contraction of the rig and nozzle.

\section{Flow and Acoustic Diagnostic Techniques}

At both GDTL and NASA, time-resolved pressure was measured using pressure transducers within the jet to explore the growth and decay of the imparted perturbations by the actuators and the ensuing instability waves/large-scale structures. Single-point static pressure and multi-point stagnation pressure, respectively, were measured at GDTL and NASA. Far field acoustic measurements were also carried out both at GDTL and NASA. In addition, PIV measurements were carried out at GDTL. Further details on these measurements are given below.

At GDTL, the axis of the Kulite (Kulite Semiconductor Products, Inc.) pressure transducer probe was normal to the jet axis so that time-resolved static pressure - rather than total pressure-was measured. The probe tip was located at the lip-line of the nozzle radially, at the center of actuator 7 (fig. 3) azimuthally, and traversed in the streamwise direction manually from $x / D=0.5$ to 7.5 . The signal from the probe was amplified, band-pass filtered, and stored on a PC. An average spectrum was obtained from one hundred short-time spectra for each case and the perturbation level at the forcing frequency was calculated from the average spectrum. For the baseline case, the level was obtained at the frequencies matching the forcing frequencies. The perturbation level was normalized by $2 \times 10^{-5} \mathrm{~Pa}$ in all cases.

Multi-point time resolved pressure measurements were recorded at NASA using an array of Endevco pressure transducers. The array used 16 pressure transducers (8 50 psia and 850 psig transducers alternating in the azimuthal direction) mounted on a ring such that the each transducer was located at the nozzle lip-line and spaced $22.5^{\circ}$ azimuthally from the previous transducer. The array was mounted on a traverse and moved in the streamwise direction from $x / D=0.4$ to 6.0 , where the flow threatened to

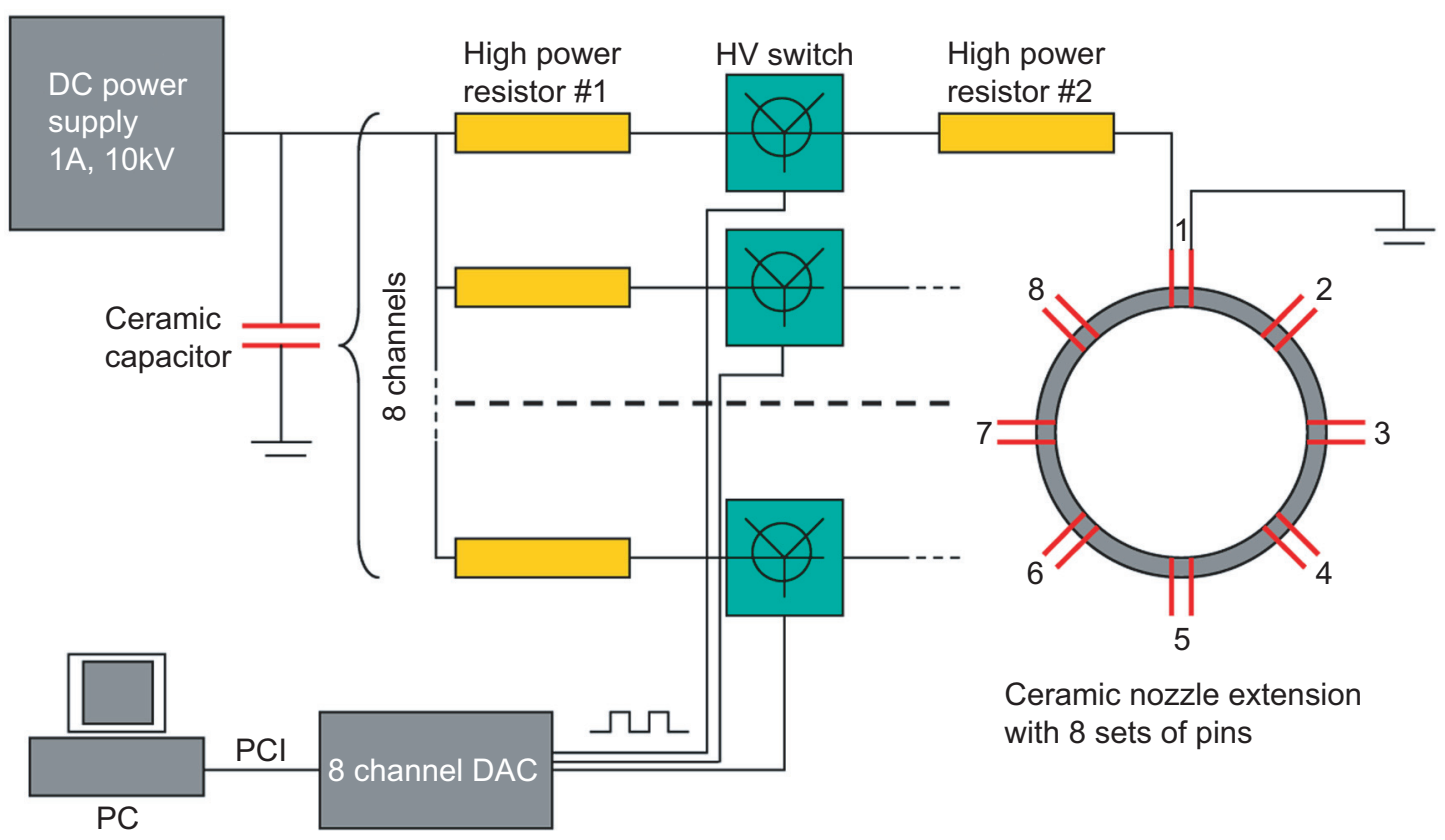

Figure 3.-Schematic of the in-house fabricated plasma generator. 
damage the array. Endevco amplifiers removed the DC signal and provided initial signal amplification. Precision amplifiers were used for further signal amplification. A 16-bit DataMax recording system, built by R.C. Electronics, digitized the data at $200 \mathrm{kHz}$ (using a $190 \mathrm{kHz}$ anti-aliasing filter). Ten seconds of continuous data were recorded for each jet condition allowing approximately 485 spectral averages based on a sample size of 8192 points and 50 percent sample overlap.

At GDTL, far field noise was measured by using two 0.25 in. Bruel \& Kjaer microphones, located 83 and 45 nozzle exit diameters from the nozzle exit at $30^{\circ}$ and $90^{\circ}$ relative to the downstream jet axis, respectively. The far field acoustic results were normalized to a radius of $80 \mathrm{D}$. The acoustic signal from each microphone was conditioned and amplified by a four-channel Bruel \& Kjaer Nexus conditioning amplifier. The signal was sampled at $200 \mathrm{kHz}$ per channel by an NI A/D board and recorded on the hard disk. As in the Kulite data processing, an average spectrum was obtained from one hundred short-time spectra. With a sample size of 8192 points, the frequency resolution is $24.4 \mathrm{~Hz}$.

At NASA, far-field noise was measured using an overhead array of twenty-four $1 / 4$-in. Bruel \& Kjaer microphones, located on an arc 72 nozzle diameters away. The arc spanned polar angles from $135^{\circ}$ to $20^{\circ}$ relative to the downstream jet axis. The microphone signals were conditioned and amplified by six units of four-channel Bruel \& Kjaer Nexus conditioning amplifiers before being digitized at $190 \mathrm{kHz}$ by a dedicated 16-bit DataMax recording system built by RC Electronics. Due to electronic noise produced by the plasma actuator delivered to the data recorder on signal lines intended to record the actuator timing, only the first $10 \mathrm{~ms}$ of data was reliable in most cases, limiting the frequency resolution of the spectra available for comparison with GDTL. The uncertainty of the spectral data is also increased to $\pm 0.7 \mathrm{~dB}$.

At GDTL, particle imaging velocimetry (PIV) was used to measure the $\mathrm{x}$ and $\mathrm{y}$ components of velocity on the $x-y$ plane (fig. 1). Images were acquired and processed using a LaVision PIV system with a 2000 by 2000 pixel Redlake CCD camera employing a 75 to $300 \mathrm{~mm}$ Vivitar zoom lens with a $532 \mathrm{~nm}$ narrow band optical filter. Other essential hardware and software are housed in a dedicated computer with dual Intel Xeon processors. The system triggers a dual-head Spectra Physics PIV-400 Nd:YAG laser operating at the $2 \mathrm{nd}$ harmonic $(532 \mathrm{~nm})$. Image pairs were acquired at a sampling rate of approximately $5 \mathrm{~Hz}$. The jet flow was seeded with DEHS fluid introduced upstream of the stagnation chamber by a fourjet atomizer. This location was chosen to provide homogenous dispersion of the particle seed throughout the jet. The ambient was seeded using a fog generator. The fog was injected into a 15-in.-diameter co-annular jet and a small amount of air flow was introduced to generate a very low speed co-flow. The camera views the streamwise laser sheet orthogonally over about 9 jet diameters. The time separation between laser pulses was $2.0 \mu$ s for images divided into 32 by 32 pixel interrogation windows. Subregions for each image pair were cross-correlated using multi-pass processing with a 50 percent overlap in order to improve spatial resolution and prevent the appearance of spurious vectors by adaptively improving the window size. Initial passes used 64 by 64 pixel interrogation windows which were then used as a reference for the 32 by 32 pixel windows in the final pass. This experimental setup produced a velocity vector grid of 115 by 65 over the measurement domain. This translated to each velocity vector being separated by approximately $2 \mathrm{~mm}$.

\section{Plasma Actuators and Plasma Generator System}

Each actuator consists of a pair of pin electrodes. The electrodes are distributed around the nozzle perimeter, approximately $1 \mathrm{~mm}$ upstream of the nozzle exit plane. A ring groove of $0.5 \mathrm{~mm}$ deep and $1 \mathrm{~mm}$ wide was used to house the electrodes and to shield and stabilize the plasma. In our earlier experiments, the plasma was swept downstream by the flow without such a groove. We have used various nozzle extensions attached to the nozzle exit to house the electrodes, as well as various types and sizes of electrodes (Samimy et al. 2004 and 2005). For the work at GDTL and NASA presented here, the nozzle extension was made of boron nitride and steel wires or tungsten wires of $1 \mathrm{~mm}$ diameter were used for electrodes. Tungsten wires have proven to be more resistant to the erosion caused by the arc discharge. Measured center-to-center, the spacing between a pair of electrodes for each actuator is $3 \mathrm{~mm}$, and the 
8 actuators at GDTL and 32 actuators at NASA were uniformly distributed around the nozzle exit. Figure 3 shows a schematic of the actuator arrangement used at GDTL.

Figure 3 also shows a schematic of the multi-channel high-voltage plasma generator, designed and built in-house, used at GDTL. The plasma generator enables simultaneous powering of up to eight localized arc filament plasma actuators distributed around the perimeter of the ceramic nozzle extension, with independent frequency, duty cycle, and phase control of individual actuators. Each actuator is connected in series with a fast response, high repetition rate, high-voltage MOSFET switch (Behlke Electronic $\mathrm{GmbH}$ ), two approximately $15 \mathrm{k} \Omega$ high power solid body ceramic ballast resistors, and a highvoltage, high-current (10 kV, 1A) DC power supply (Glassman High Voltage, Inc.). Two of these power supplies are used to energize eight actuators. If all eight actuators are powered at the same time, the single actuator current is limited to $0.25 \mathrm{~A}$. The switches are controlled by using an 8-channel digital-to-analog output PCI card and the LabView software, which allows their independent frequency, duty cycle, and phase control. The switches are capable of producing high voltage pulses (up to $10 \mathrm{kV}$ ) at repetition rates from 0 to $200 \mathrm{kHz}$, with a very short pulse rise/fall time $(\sim 0.1 \mathrm{~s})$ and a variable duty cycle (from 0 to 100 percent). Every switch is liquid cooled to allow continuous operation at high frequency, voltage and current.

By turning the electronic switch on and off, positive high voltage pulses can be applied to the corresponding actuator. The high initial voltage is needed to produce breakdown in the approximately atmospheric pressure air in the gap between the two electrodes of an actuator, which are $3 \mathrm{~mm}$ apart in the present work. After the breakdown, the arc is generated and the voltage across the gap rapidly falls to a few hundred volts. The plasma generator is compact, robust, and simple to operate. In the present work, continuous operation of all eight actuators in an $M=0.9$ flow (up to several minutes) has been achieved. To reduce the EMI interference with the computer and data acquisition board, an in-house built optical isolation circuit was implemented in the low voltage side.

In a typical acoustic driver, which has been used extensively in the literature for flow control, the input signal is a sine wave of prescribed frequency, peak amplitude and phase. The input amplitude changes gradually between the given positive and negative peaks in a cycle. On the other hand, the input signal to a plasma actuator is a rectangular wave with a variable duty cycle (for more details, see Kastner et al., 2006). In a plasma actuator, the input or forcing amplitude cannot easily be altered, as in an acoustic driver. Although the imparted forcing energy to the flow in a cycle can be adjusted by adjusting duty cycle, the effective forcing amplitude is not directly related to the total energy in a cycle. However, it is straightforward to force the jet in any simple azimuthal mode $(m=0,1,2,3, \ldots, \mathrm{N} ; \mathrm{N}$ depending on the number of actuators used) since there is no forcing amplitude variation. For combined/mixed azimuthal modes $(m= \pm 1, \pm 2, \ldots)$, the input amplitude is the modulated amplitude of the positive and negative waves. These modes were simulated or mimicked by grouping the actuators without any amplitude modulation, which is not possible with the plasma actuators. For example, the top three actuators $(1,2$, and 8 ) and the bottom three actuators (4 to 6) in figure 3 were grouped together and operated $180^{\circ}$ out of phase to simulate $m= \pm 1$ mode. For this mode, actuators 3 and 7 were not operated to make these positions nodal points of $m= \pm 1$ mode. For other mixed mode, a similar grouping was done. The focus of this paper is on forcing jet instabilities using these actuators. There are two additional secondary effects. First, the actuator input signal has a prescribed frequency, but since it is a rectangular wave, it contains higher frequencies as well. In addition, each actuator would perhaps generate a pair of very weak streamwise vortices. While we plan to explore the second issue in the near future, any potential effects of the first subject, perceived to be small, is difficult.

At NASA, the nozzle exit diameter was $19.05 \mathrm{~cm}$ (7.5 in.), which is 7.5 times the jet diameter at GDTL. To partially account for the increased diameter, we used 32 actuators uniformly distributed around the nozzle. A simple linear scaling-up would have required 60 actuators. But the available power supply could only power 8 actuators at a time. Therefore a decision was made to use 32 actuators equally distributed around the nozzle, enabling us to force the jet only with azimuthal modes $m= \pm 1,4$, and 8 . For this arrangement, additional $48 \mathrm{HV}$ resistors were added to the generator and the wiring was 
significantly modified to run larger number of actuators so that each transistor was able to switch 4 actuators at the same time.

\section{Experimental Results}

The focus of the experimental results will be on forcing over a large frequency range high subsonic jets $\left(M_{a}=0.5\right.$ to 0.84$)$. The forcing will be mostly with azimuthal mode $m= \pm 1$ with limited results from $m=4$ and 8 forcing.

\section{Plasma Actuator Input Characterization}

Figure 4 shows time-dependent voltage, current, and power in the arc discharge between two steel wire electrodes of a plasma actuator taken at GDTL. During these measurements, only one actuator was operated at $20 \mathrm{kHz}$ frequency and a 20 percent duty cycle in the ideally expanded $M=1.3$ jet. Results in $M=0.9$ jet are similar. The static pressure at the actuators location $(1 \mathrm{~mm}$ before the nozzle extension exit) was approximately $1 \mathrm{~atm}$. The top pulse train is the input signal to the high-voltage switch. The second graph from the top shows time-dependent voltage between the actuator electrodes, measured at point 1 (see fig. 3) by a Tektronix high voltage probe P6015A. A very large voltage overshoot (about $4.0 \mathrm{kV}$ ) at the onset of the cycle ( $\sim 12 \mu \mathrm{s}$ on the graph) corresponds to electrical breakdown in the air
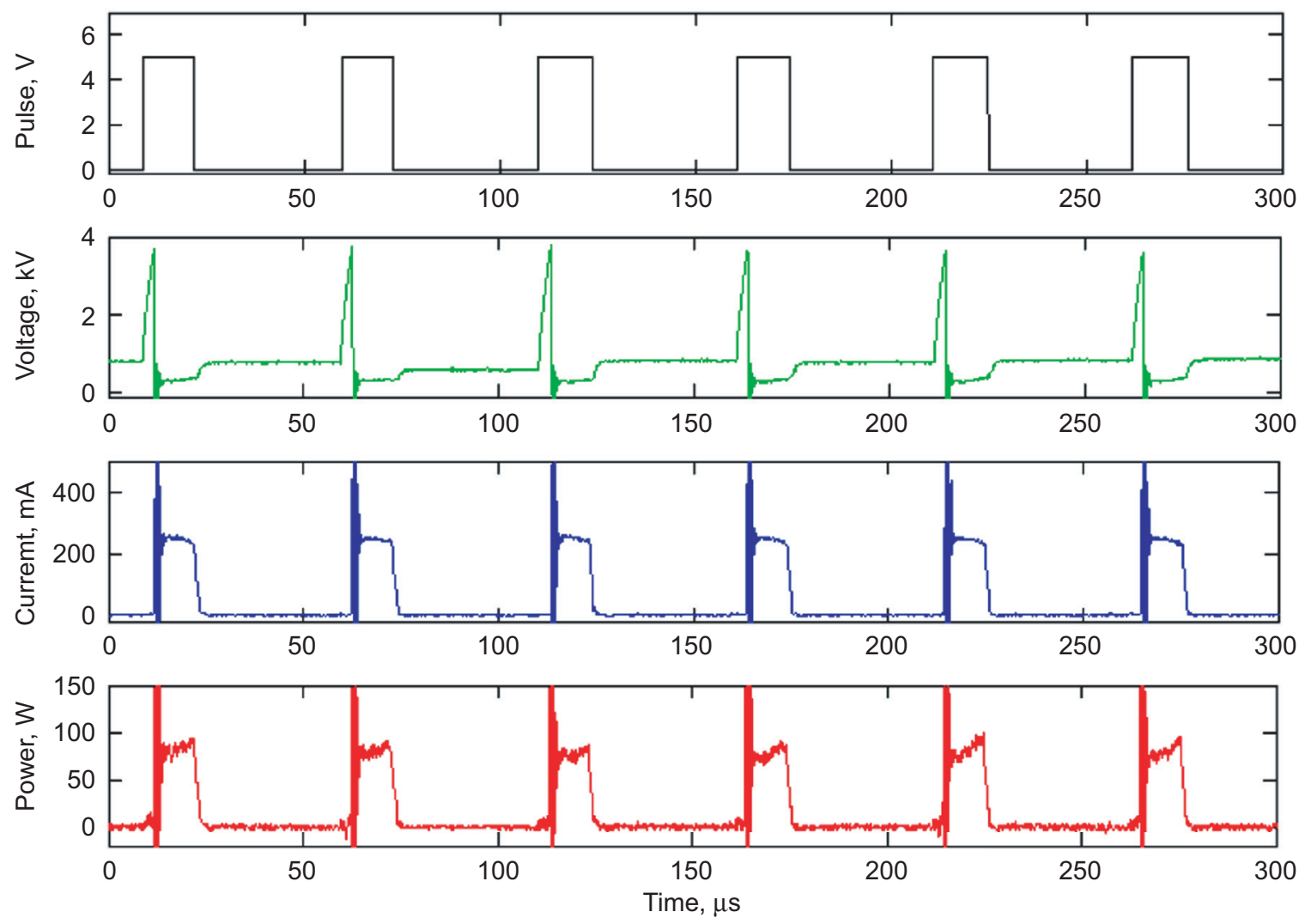

Figure 4.-Time-dependent voltage, current, and power in a plasma actuator operated at $20 \mathrm{kHz}$ frequency and 20 percent duty cycle (only one actuator was operated). The top pulse train is the input signal to the high-voltage switch. 
between the electrodes, followed by a sharp voltage drop to about $400 \mathrm{~V}$, as a stationary arc discharge is formed. After the discharge current is interrupted by rapidly closing the switch (10 $\mu$ s later), the plasma is quickly blown off by the flow. As a result, the high voltage electrode (anode) becomes floating and the voltage remains relatively low (at about 600 to $700 \mathrm{~V}$ ) until the moment the switch is opened again $50 \mu \mathrm{s}$ later.

The actuator current was measured simultaneously with the voltage measurement using a LeCroy CP031 current probe and is shown in figure 4. The current trace (third from top) is nearly a step function, with the steady state current of about $250 \mathrm{~mA}$ when the switch is open and no current when it is closed. The time-dependent arc discharge power can be obtained simply by multiplying the current and voltage traces. The result is shown in the bottom graph of figure 3 . The power during the current pulse reaches approximately $100 \mathrm{~W}$, which corresponds to a time-averaged actuator power of only $20 \mathrm{~W}$ (i.e., $160 \mathrm{~W}$ net power for all eight actuators in operation). For comparison, the flow power (the total enthalpy flux) of the jet at these conditions is about $28 \mathrm{~kW}$. These results demonstrate that high-speed flow control by localized arc plasma actuators can be highly energy efficient.

In an effort to understand the mechanism behind such efficiency we have conducted a series of plasma temperature measurements. The temperature was measured by monitoring the emission of second positive band system of nitrogen from the arc region. The emitted light was directed through an optical fiber to a Princeton Instrument Optical Multi-channel Analyzer, which was equipped with an ICCD camera. Least squares fit was used to compare the experimental spectra of nitrogen second positive system and the synthetic spectra generated by a theoretical code with temperature being a variable parameter. The results show that the gas temperature rises extremely fast after the breakdown and reaches $1700{ }^{\circ} \mathrm{C}$ in several microseconds. This rapid heating might be the key for the actuator's dramatic influence on the flow that has been demonstrated in our experiments.

The measurements also showed that the voltage, current, and power traces for all eight actuators are essentially the same. Depending on the frequency, mode, and duty cycle the arc discharges produced by different actuators may overlap in time. The same is true about the 32 actuators that we ran at NASA facility. The traces look very similar to those in figure 4, except that the duty cycles were kept very low (usually 1 to 3 percent) because of much of much lower forcing frequencies at NASA's larger facility.

\section{Growth and Decay of Perturbations and the Ensuing Instability Waves}

The perturbation caused by the plasma actuators in the jet shear layer was measured both in the OSU and NASA facilities. The measured pressure is static at GDTL, while it is total at NASA. At GDTL, detailed analysis has been done in the $M_{a}=0.84$ jet in order to explore the growth and decay of the perturbation seeded into the flow by plasma actuators and the ensuing instability waves. The pressure transducer was located at the same azimuthal location as actuator number 7 in figure 3, was traversed in the streamwise direction from $x / D=0.5$ to 7.5. The tip of the transducer grazes the shear layer at the first measurement location $(x / D=0.5)$, but is well into the shear layer in farther downstream locations, as the shear layer is growing. The amplitude of the local hydrodynamic pressure at the forcing frequency was obtained using power spectrum of the time-resolved pressure signal.

The spatial development of the perturbation level for the GDTL jet is shown in figures 5 and 6 for the flapping and non-flapping plane, respectively. At all $\mathrm{St}_{\mathrm{DF}}$, the maximum perturbation level is about the same on the flapping plane. The growth rate of the perturbation is slower at a lower forcing frequency and the saturation occurs at a farther downstream location when it is compared to a higher frequency forcing case (fig. 5). When the jet was forced at $S t_{D F}=0.36$, which is in the jet preferred frequen

cy range, saturation occurred at $x / D=2.5$, but the fluctuation level remained relatively high to a farther downstream location. This growth and decay is consistent with the flow visualization in an ideally expanded Mach 1.3 jet (Samimy et al. 2005), where the large-scale structures grew in size until they reach the end of potential core. As the forcing frequency increases, the saturation position is getting closer to the jet exit and the perturbation level decays very rapidly after saturation. It seems that the maximum perturbation level was reached upstream of the first measuring location of $x / D=0.5$ for $S t_{D F}=1.81$. 


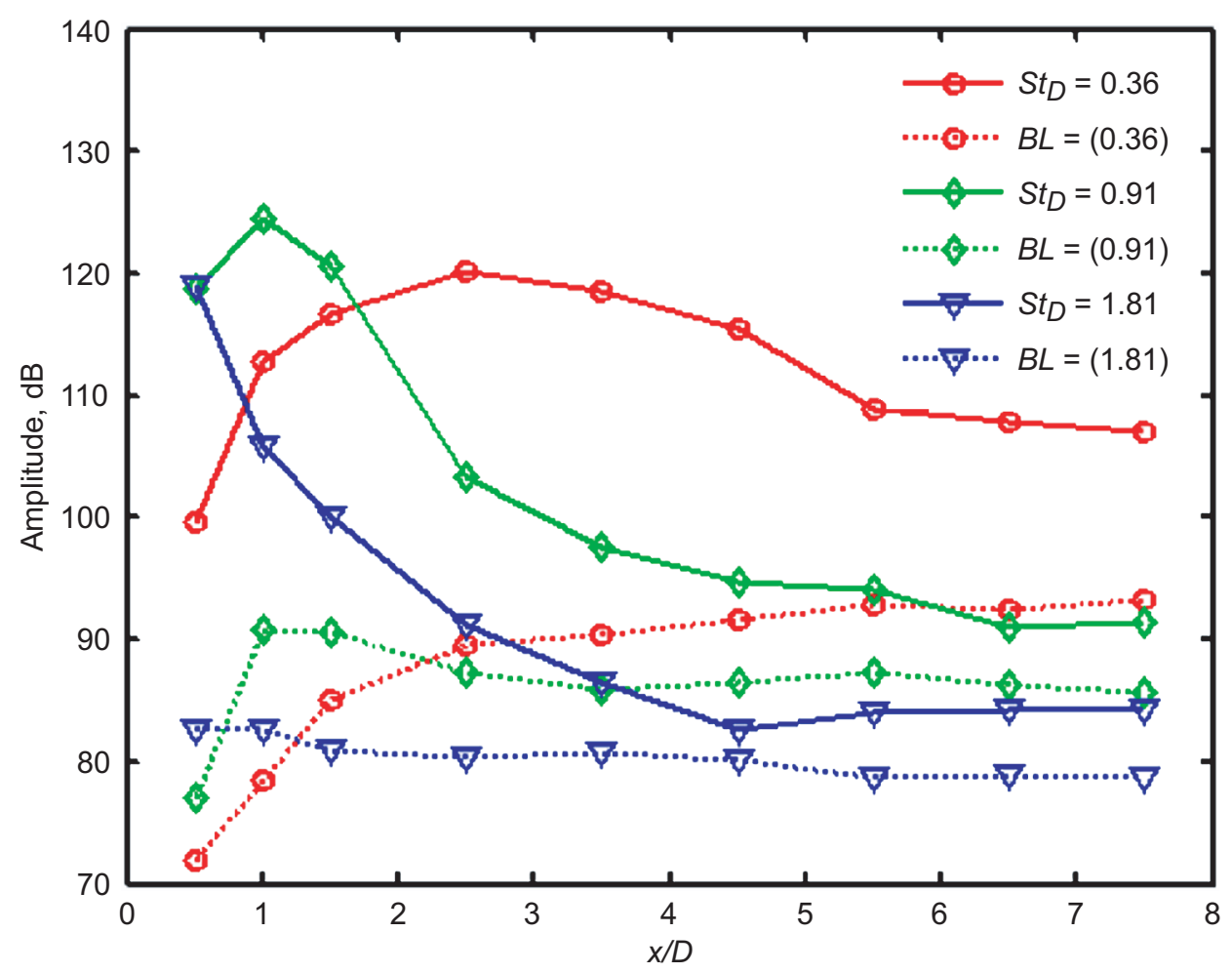

Figure 5.-Spatial development of seeded perturbations on the flapping plane at several Strouhal numbers $\left(S t_{D F}\right)$ for azimuthal mode $m= \pm 1$.

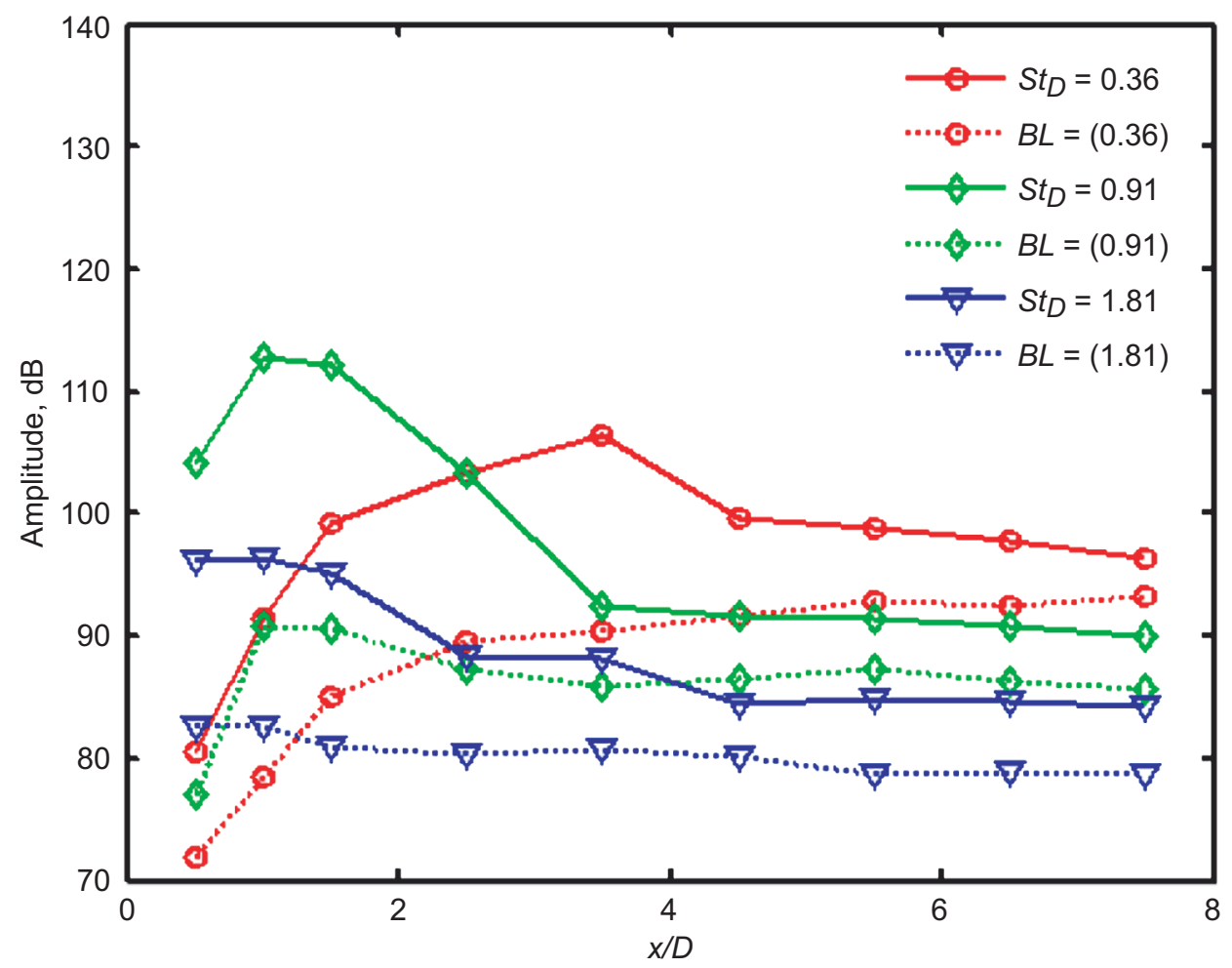

Figure 6.-Spatial development of seeded perturbations on the non-flapping plane at several Strouhal numbers $\left(S t_{D F}\right)$ for azimuthal mode $m= \pm 1$. 

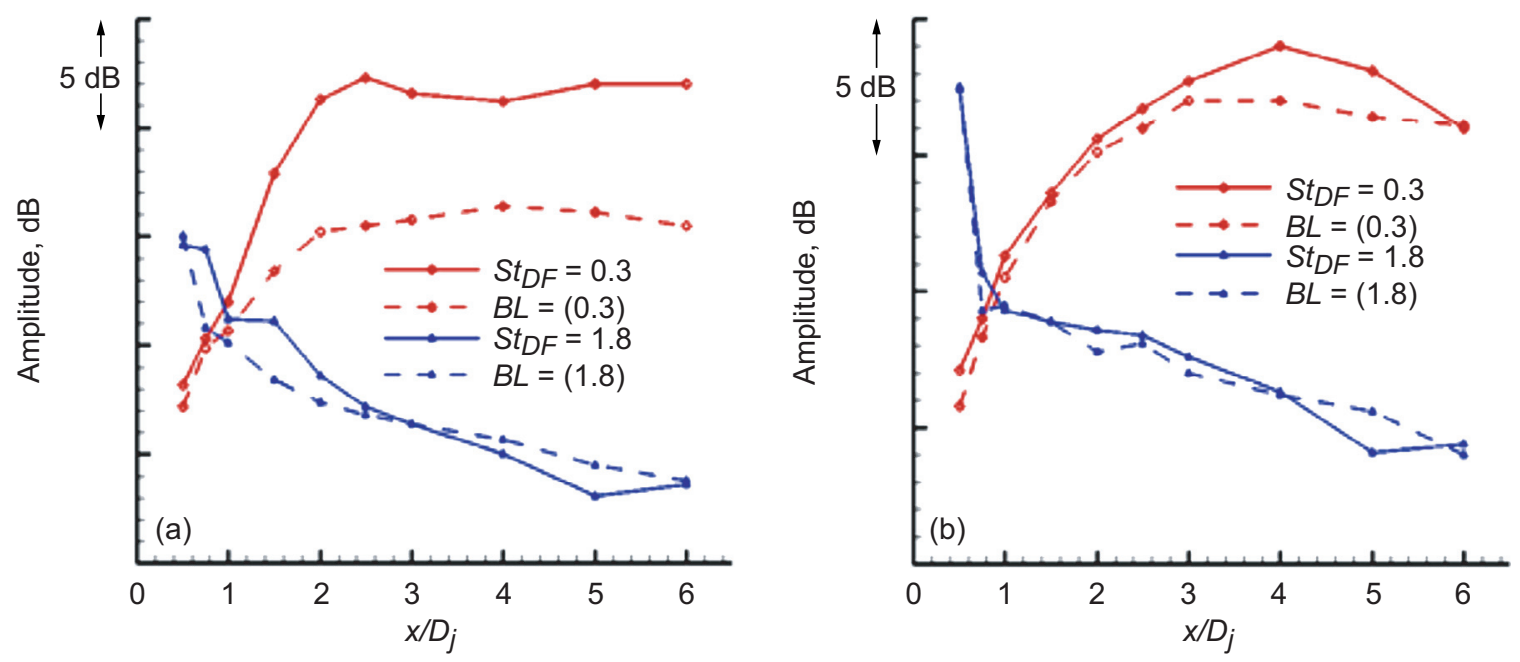

Figure 7.-Axial growth of input perturbations in the flapping (a) and non-flapping (b) plane at a $M_{a}=0.5$ jet condition perturbed in the azimuthal \pm 1 mode.

When the jet is excited at a higher frequency, the flow structures are smaller and they decay quickly as seen in Samimy et al. (2005). This explains why the perturbation level decays rapidly at higher forcing frequencies.

On the non-flapping plane, the maximum fluctuation level is 10 to $25 \mathrm{~dB}$ lower than that on the flapping plane. This lower fluctuation level was expected since the probe was on the nodal point of the flapping motion. Although the perturbation level was reduced, the amplification trends appear to be similar to those observed on the flapping plane.

The axial growth of the input perturbations measured at NASA is shown in figure 7 for the flapping and non-flapping planes, at a $M_{a}=0.5$ jet condition. Only perturbation frequencies of $S t_{D F}=0.3$ and $S t_{D F}=1.8$ were considered during this phase of NASA testing due to time constraints. In the flapping plane, at a perturbation frequency $S t_{D F}=0.3$, near the jet preferred frequency, the perturbed jet shows a significant amplitude increase compared to the baseline jet. The saturation point for the perturbed jet appears between $x / D=2$ and $x / D=3$ and is followed by a slow decay. In the non-flapping plane, the perturbed jet again saturates at a higher amplitude than the baseline jet and at $x / D=40$, slightly downstream of the jet observed in the flapping plane.

The $M_{a}=0.5$ jet perturbed at $S t_{D F}=1.8$ shows limited response in the flapping plane close to the nozzle exit (less than $x / D=2$ ). No significant response is observed further downstream in the flapping plane or at any axial location in the non-flapping plane. The NASA jet appears to be fairly insensitive to this forcing frequency.

The spatial development of the input perturbations were also recorded at NASA using a $M_{a}=0.84$ jet condition (fig. 8) to compare against the detailed data recorded at GDTL. Results in the flapping plane show the jet is responding to the perturbations based on comparison with the baseline. Saturation of the excited mode occurs around $x / D=5$, where the excited jet is $3 \mathrm{~dB}$ above the baseline. Data in the nonflapping plane also shows increased amplitude in the jet excited at $S t_{D F}=0.3$ versus the baseline, but, as expected, the difference is smaller. The saturation peak in the non-flapping plane appears much broader, occurring somewhere $x / D=3$ and $x / D=5$. In both the flapping and non-flapping planes, the amplitude difference between the excited and unexcited jet is smaller and the saturation point occurs further downstream in NASA data than was observed in the GDTL data. No significant response is observed when the jet is perturbed at $S t_{D F}=1.8$, again showing that the NASA jet, unlike the GDTL jet, is not particularly sensitive to this perturbation frequency. 

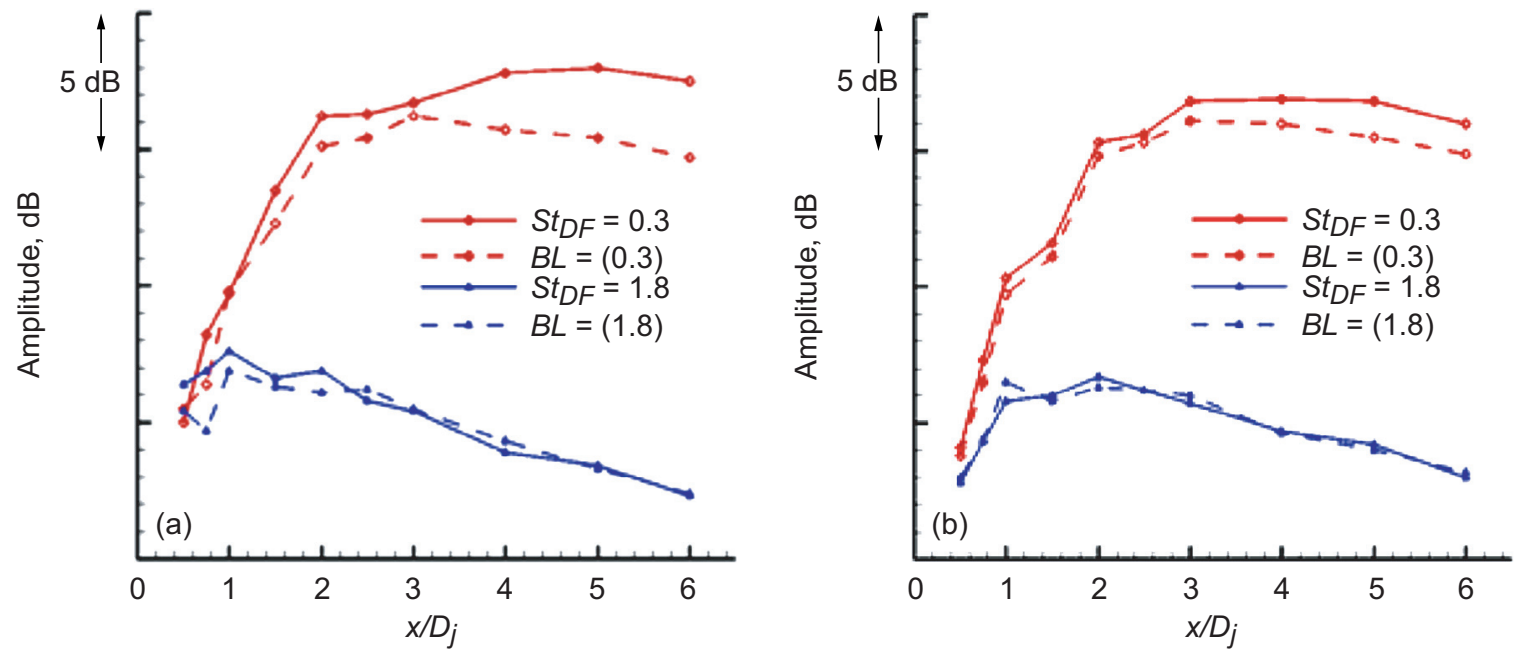

Figure 8.-Axial growth of input perturbations in the flapping (a) and non-flapping (b) plane at a $M_{a}=0.9$ jet condition perturbed in the azimuthal \pm 1 mode.

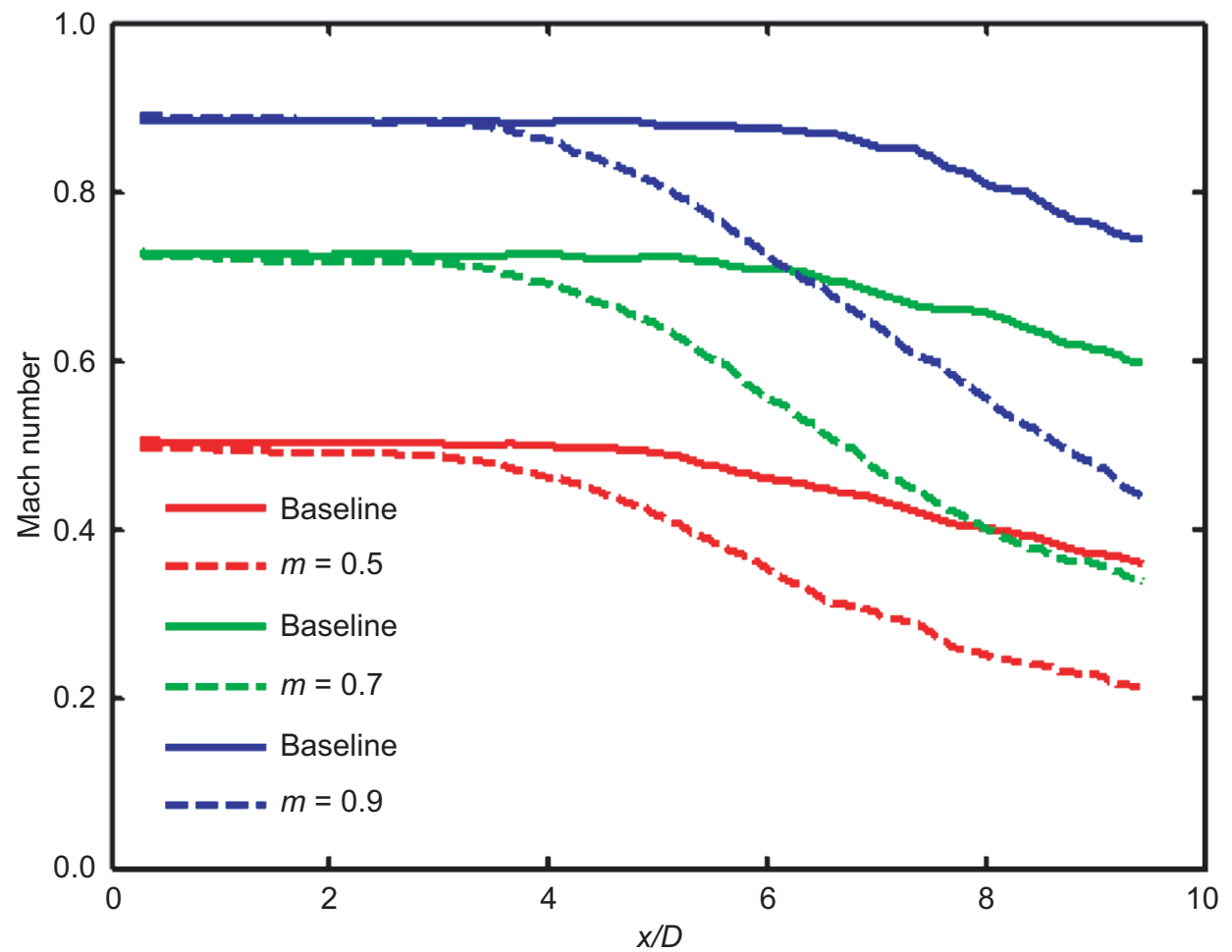

Figure 9.-Centerline Mach number for various jet Mach numbers with forcing Strouhal number of $\sim 0.3$ and azimuthal mode $m= \pm 1$.

\section{Flow Results}

PIV measurements on a streamwise plane passing through the centerline of the jet (fig. 1) were carried out at GDTL at three $M_{a}=0.5,0.7$, and $0.84(M=0.51,0.74$, and 0.9$)$. The measured centerline Mach number profiles are shown in figure 9 for the baseline cases as well as forced cases with a Strouhal number of approximately 0.3 (the actual forcing Strouhal numbers are 0.31, 0.33, and 0.27 for the acoustic Mach numbers 0.5, 0.7, and 0.84, respectively). These Strouhal numbers are close to the jet 


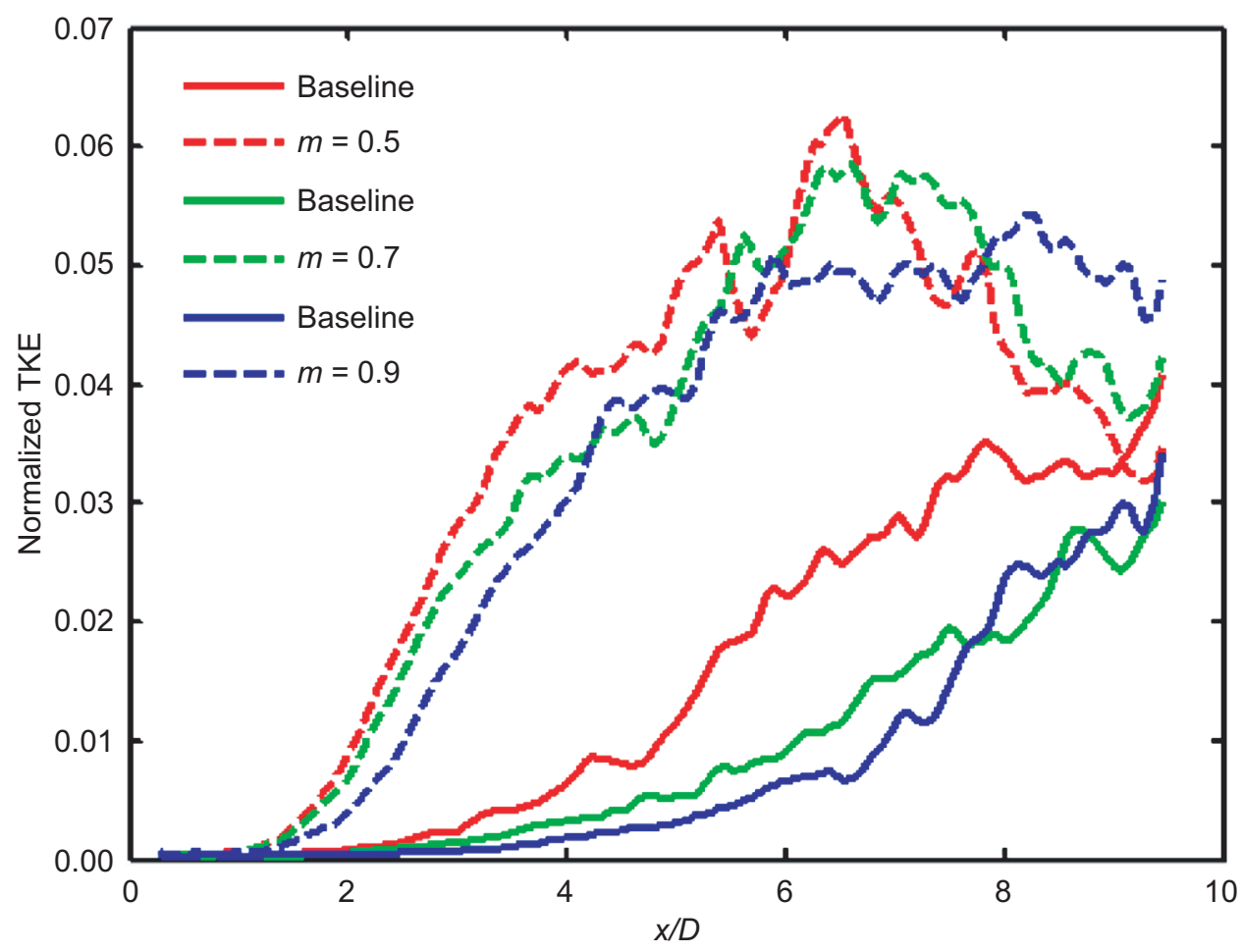

Figure 10.-Centerline turbulent kinetic energy for various jet Mach numbers with forcing Strouhal number of $\sim 0.3$ and azimuthal mode $m= \pm 1$.

preferred Strouhal numbers, based on the jet centerline Mach number measurements. In all cases, the forcing amplitude remained the same, as there is no control on this amplitude. In the baseline cases, as the jet Mach number increases, the potential core becomes longer: $L_{p} / D=5.0,6.1$, and 6.5, respectively, for the jet $M_{a}=0.5,0.7$, and 0.84 . The potential core lengths were shortened to approximately $L_{p} / D=3.0$, 3.3 , and 3.5 by the forcing for the jet $M_{a}=0.5,0.7$, and 0.84 , respectively. While the centerline Mach number decay rate downstream of the jet potential core is significantly increased for the forced cases, in comparison with the baseline cases, they are about the same for all three forcing cases. The centerline Mach number for the forced cases is about 40 percent of the corresponding baseline case at $x / D=9.0$.

The centerline turbulent kinetic energy variations for all three Mach numbers are shown in figure 10. While the baseline Mach 0.7 and 0.84 cases show similar centerline kinetic energy distribution, the values for the lowest Mach number are much higher. The cause for the difference is not clear at this time, but it could be Reynolds number effect - the boundary layer is perhaps laminar at the nozzle exit for Mach 0.5. For the controlled cases, although there are some differences for different Mach numbers, the overall effects of forcing at $m= \pm 1$ appears to be similar for all the jet Mach numbers. For all the cases, the centerline TKE increases by several folds over a significant portion of the jet.

For the jet Mach number of 0.84 and the forcing azimuthal mode of $m= \pm 1$, the forcing Strouhal number was varied from 0.1 to 3.0 to investigate the effects of forcing frequency. As shown in figure 11, the jet core shortens and the centerline Mach number decays much faster as the forcing Strouhal number approaches $S t_{D F}=0.27$ from either lower or higher side. This trend with forcing Strouhal number is similar to that for an ideally expanded Mach 1.3 jet (Samimy et al. 2006a). At this preferred (or close to preferred) Strouhal number, the centerline turbulent kinetic energy grows faster than at any other forcing Strouhal number. The centerline turbulent kinetic energy trend, shown in figure 12, is similar to that of the centerline Mach number. Forcing at the preferred mode, TKE level increased by an order of magnitude in comparison with the baseline case and with those forcing at much higher or lower Strouhal numbers. It was shown Samimy et al. (2006a), that large and specially and temporally coherent structures were generated by the forcing a Mach 1.3 ideally expanded jet with $m= \pm 1$ at the preferred mode. 


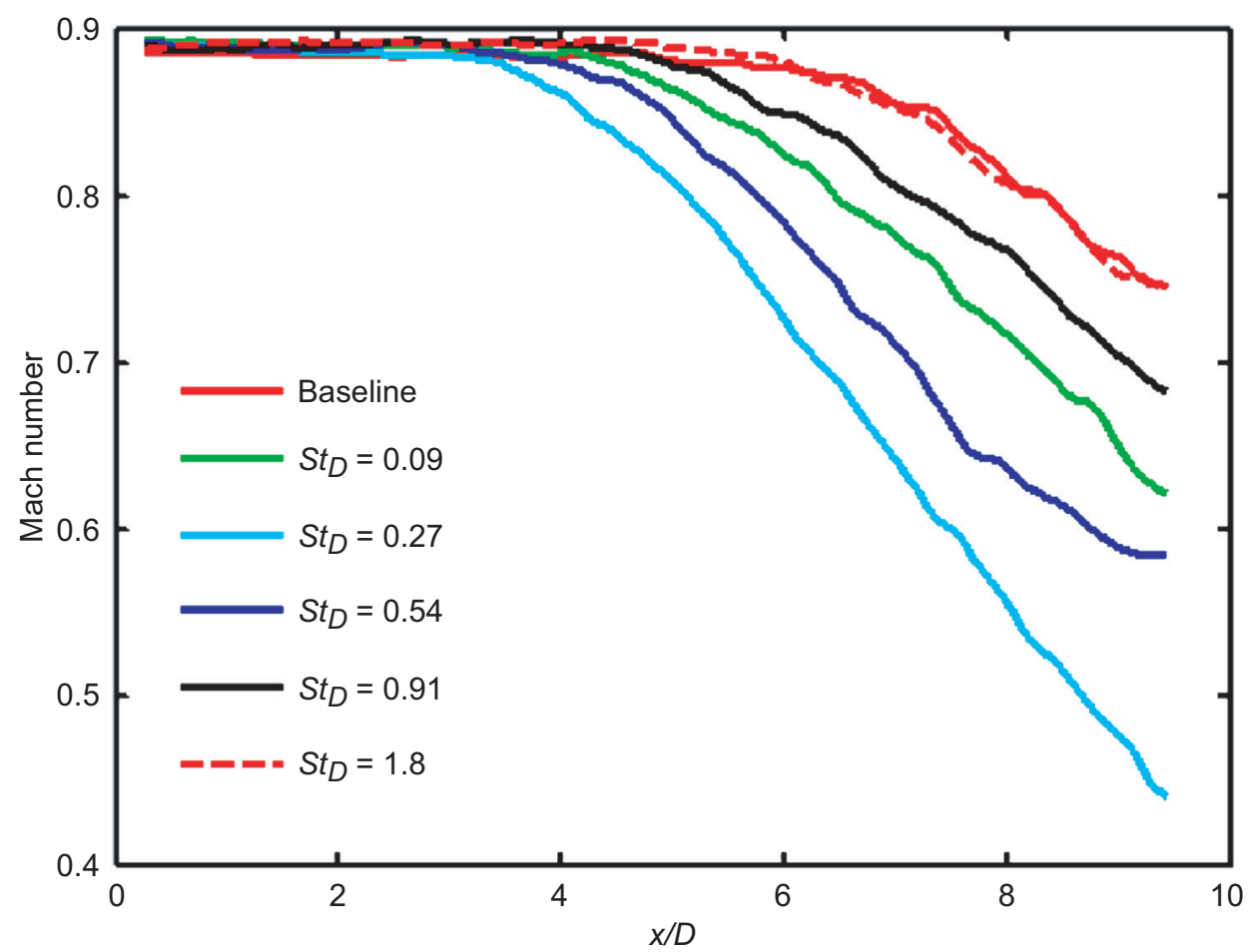

Figure 11.-Centerline Mach number for the jet acoustic Mach number 0.84 with forcing azimuthal mode $m= \pm 1$ and various forcing Strouhal number.

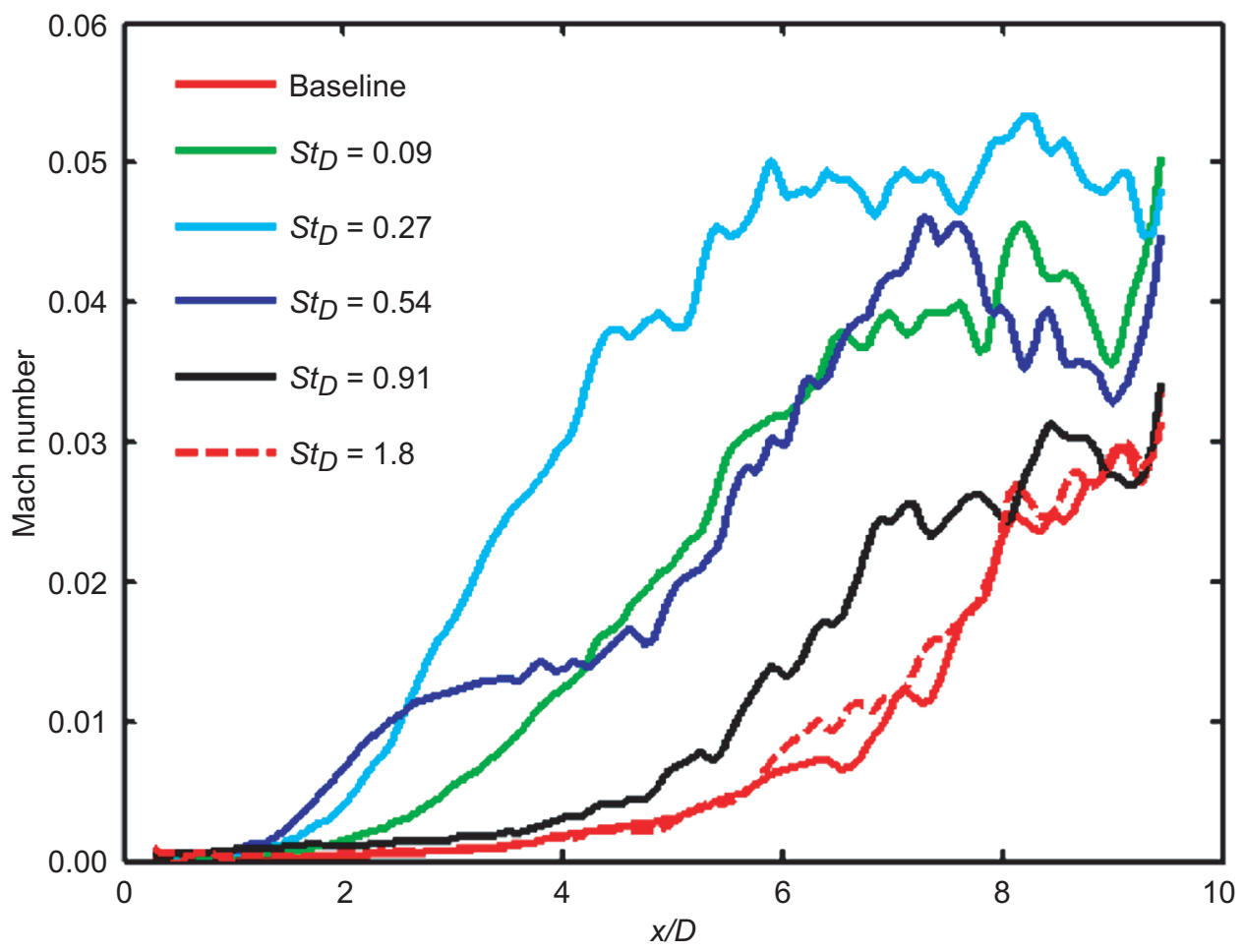

Figure 12.-Centerline turbulent kinetic energy forthe jet acoustic Mach number 0.84 with forcing azimuthal mode $m= \pm 1$ and various forcing Strouhal number. 
Although it is not possible to visualize the large-scale structures at these subsonic jets by the same techniques, it is expected that similar large-scale structures exist in these jets as well. Obviously, the periodic large-scale-structures are responsible for the entrainment mixing and thus significant increase in turbulent kinetic energy.

\section{Far Field Acoustic Results}

For the preliminary experiments at NASA we had two major constraints. First, we had to reduce the actuator number density by a factor of two ( 8 at GDTL for $1 \mathrm{in}$. nozzle and 32 at NASA for $7.5 \mathrm{in}$. nozzle). Second, we could drive only 8 of the actuators at a time. These constraints limited the azimuthal mode forcing to only $m= \pm 1,4$, and 8 . On the other hand the highest azimuthal mode we could run at GDTL was $m=3$. Therefore, we will present far field acoustic from both GDTL and NASA while forcing at various frequencies with $m= \pm 1$ and also some limited forcing cases at $m=4$ and 8 from NASA. Figures 13 and 14 show power spectra at several forcing Strouhal numbers for far field acoustic at $90^{\circ}$ and $30^{\circ}$ with respect to the downstream jet axis for acoustic Mach number 0.5 jets at GDTL and NASA, respectively. The azimuthal mode is \pm 1 and the microphones are located on the flapping plane of the jet for both cases. It appears that the small jet at GDTL at this Mach number is overwhelmed by forcing, the actuation tone and its harmonics appear in the spectrum, and noise is increased across the broadband at both angles in most of the forcing cases. The amplification is strongest when forcing close to the jet preferred mode frequency. Only in the two highest Strouhal number forcing cases $\left(S t_{D F}=1.7\right.$ and 5.0) is the broadband noise slightly reduced over a portion of the spectrum. There are some similarities and some differences between the results at GDTL and NASA. The main differences are that the jet at NASA is not overwhelmed by the actuation, and the forcing tones and their harmonics are not very visible until much higher forcing frequencies. The similarities are noise amplification in lower forcing frequencies, maximum amplification at $S t_{D} \sim 0.3$ and slight noise reduction at much higher forcing frequencies. The results from the microphones on the non-flapping plane show similar trends.
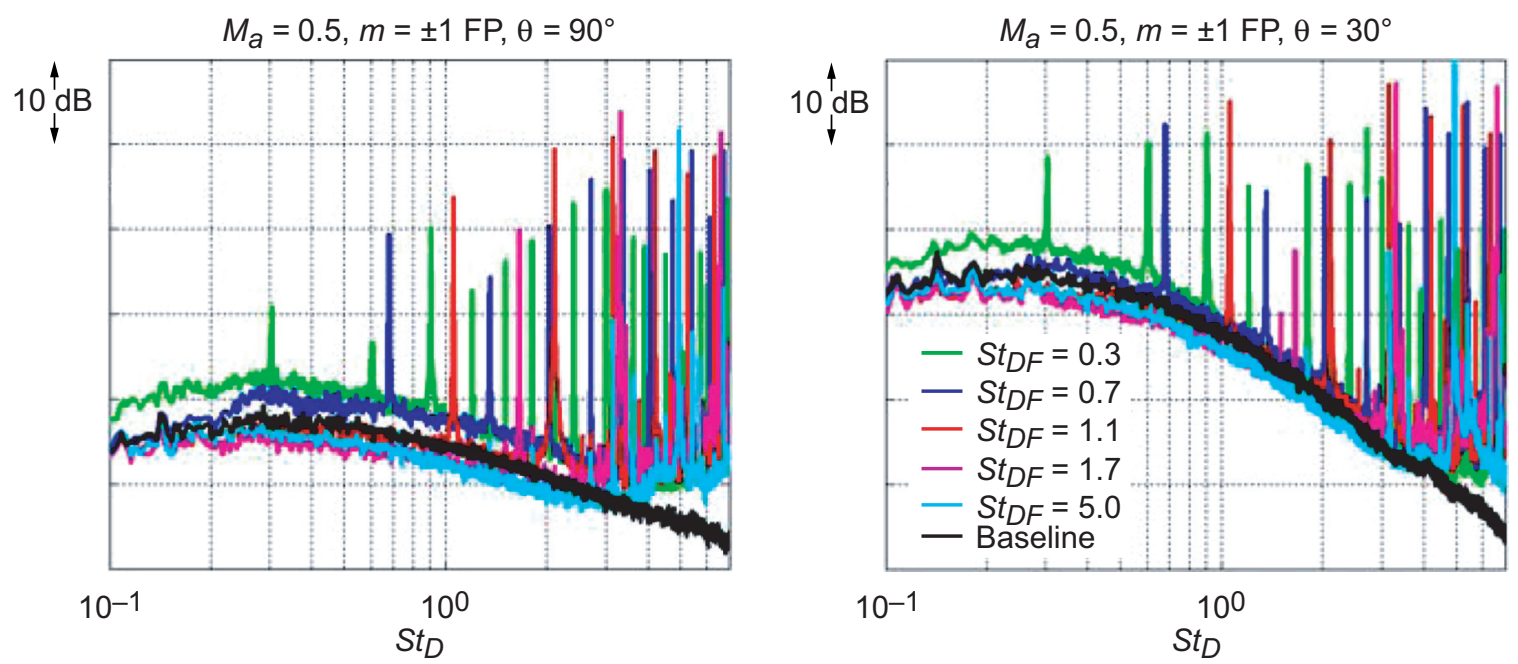

Figure 13.-Far field noise spectra for $M_{a}=0.5$ jet at GDTL for forcing at various Strouhal number with azimuthal mode $m= \pm 1$. 

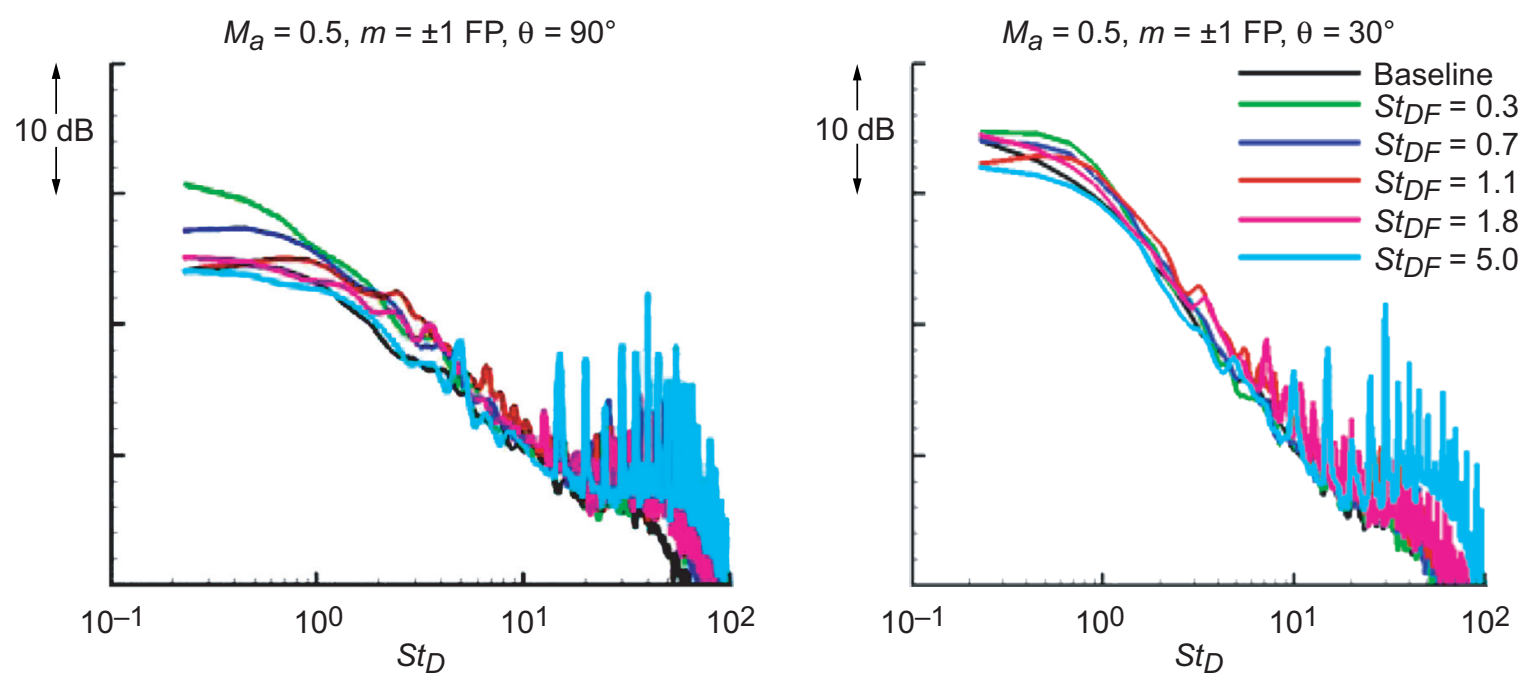

Figure 14.-Far field noise spectra for $M_{a}=0.5$ jet at NASA for forcing at various Strouhal number with azimuthal mode $m= \pm 1$.
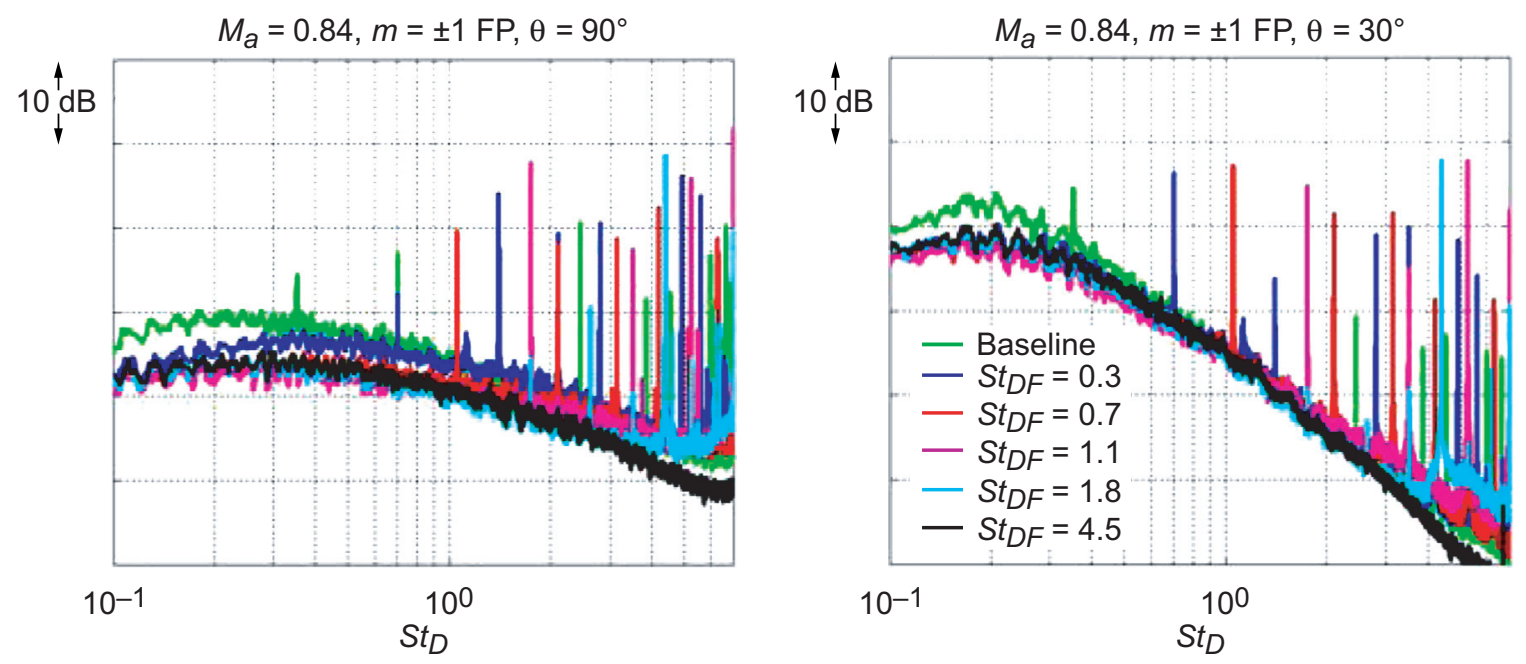

Figure 15.-Far field noise spectra for $M_{a}=0.84$ jet at GDTL for forcing at various Strouhal number with azimuthal mode $m= \pm 1$.

Figures 15 and 16 show far field noise power spectra at the same forcing Strouhal numbers, forcing azimuthal mode, observation angles and observation plane as those in figures 13 and 14, but for $M_{a}=0.84$. For GDTL (fig. 15) the results are very similar to $M_{a}=0.5$ in figure 13. For NASA, the noise is still increased when forcing at the lower $S t_{D F}$, but no longer is there any reduction when forcing the high $S t_{D F}$. As stated earlier, the number of actuators did not directly scale between the two facilities, thus the lack of actuator authority at higher Mach numbers probably points to the need for more actuators. Detailed far field acoustic results from the GDTL facility for jet $M_{a}=0.84$ were presented in Samimy et al. (2006b). Figure 17 summarizes the results seen from that paper by presenting the change in the OASPL, over the $S t_{D}$ range of 0.01 to 5.0 , when exciting over a $S t_{D F}$ range of 0.1 to 5 with various azimuthal modes. Several observations can be made which complement the results seen in figures 13 and 15 . 

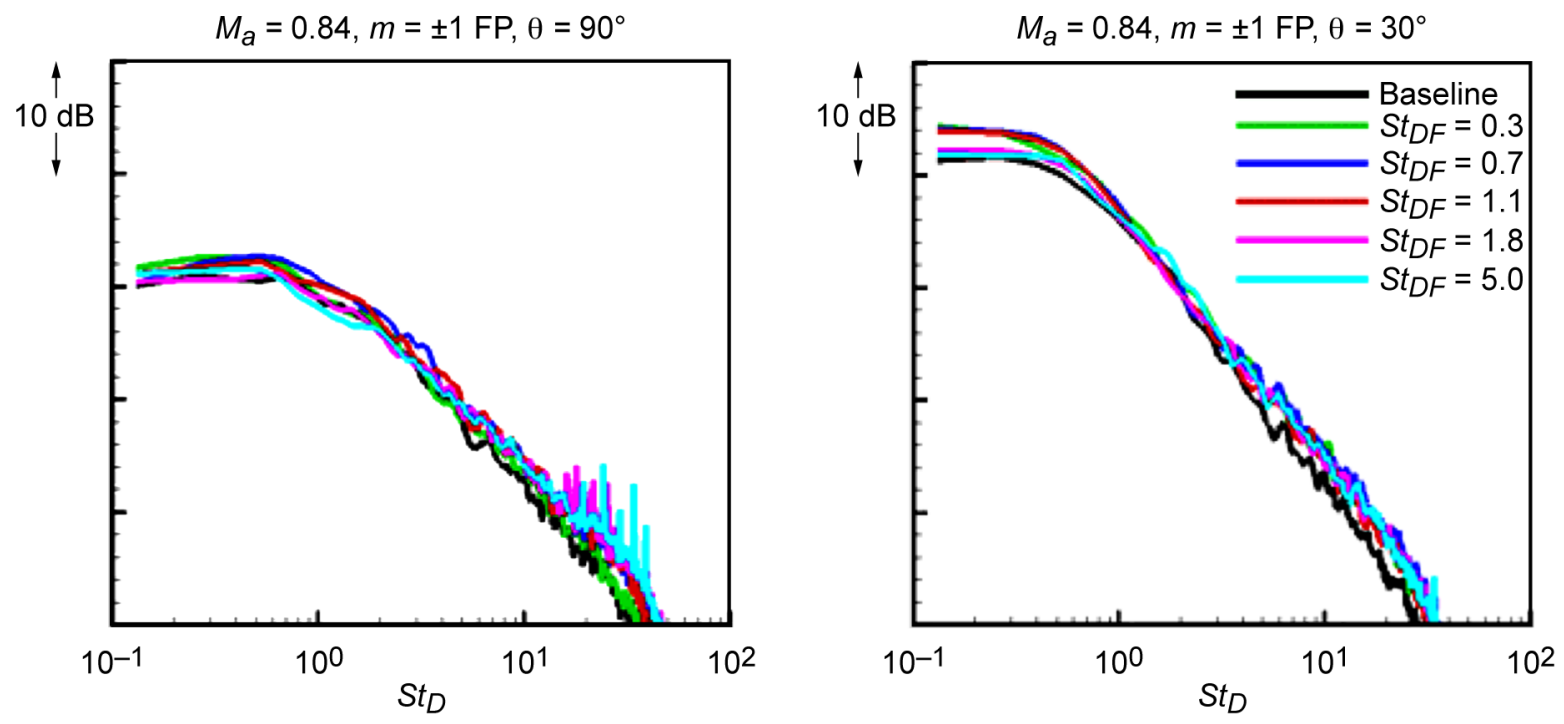

Figure 16.-Far field noise spectra for $M_{a}=0.84$ jet at GDTL for forcing at various Strouhal number with azimuthal mode $m= \pm 1$.
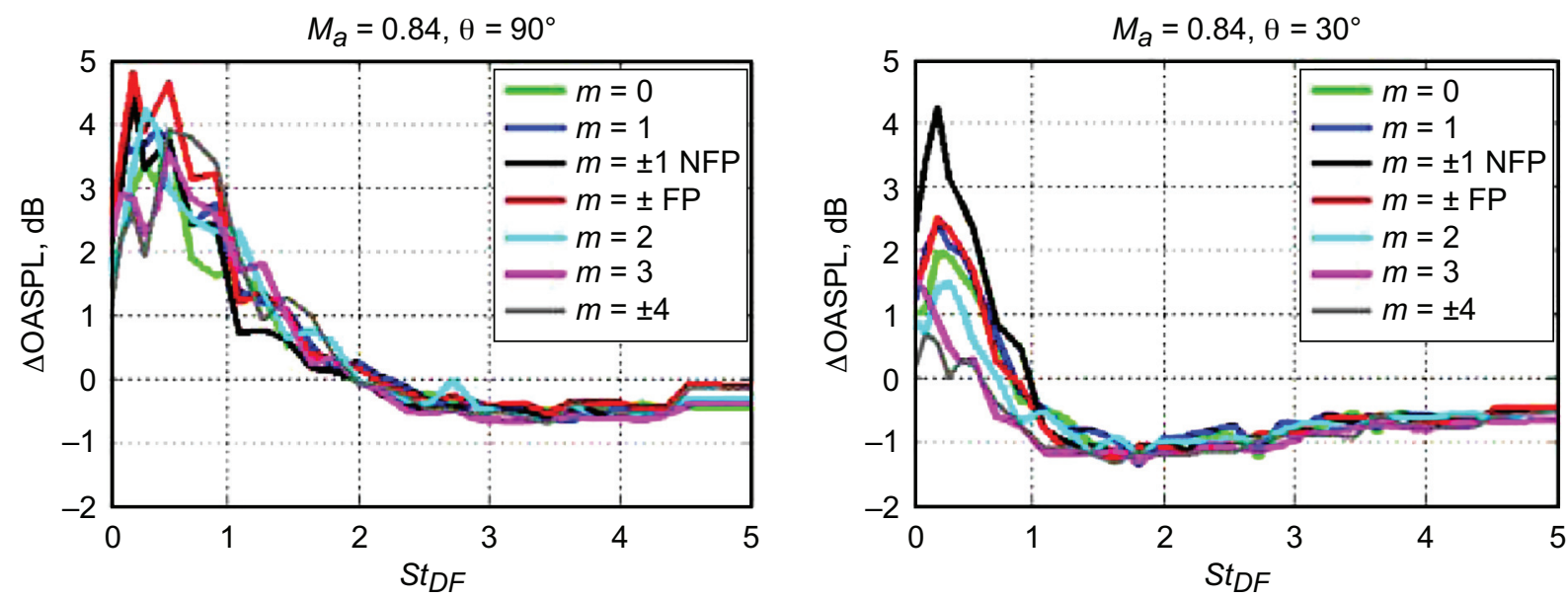

Figure 17.-Changes in the far field sound pressure level between $S t_{D F}=0.01$ and 5.0 at $90^{\circ}$ and $30^{\circ}$ over a large forcing Strouhal number $\left(S t_{D F}\right)$ range for different azimuthal modes.

(1) There is a significant noise increase when the jet is excited around the preferred frequency of the jet column instability ( $\mathrm{StDF}=0.2$ to 0.5 ). This is in agreement with the results in the literature using acoustic drivers (e.g., Jubelin, 1980; Ahuja et al., 1982; Lu, 1983).

(2) Noise reduction of 0.6 to over $1.0 \mathrm{~dB}$ is achieved over a large range of excitation frequencies. This reduction seems to peak between $S t_{D F}=1.5$ to 2.0 at $30^{\circ}$ and around $S t_{D F}=3.0$ to 3.5 at $90^{\circ}$.

(3) There seems to be a clear advantage of excitation with azimuthal modes $m=3$ and \pm 4 . These were the highest azimuthal modes that could be excited with the 8 actuators used at GDTL. However at NASA, 32 actuators were used and azimuthal modes $m=4$ and 8 could be excited. 

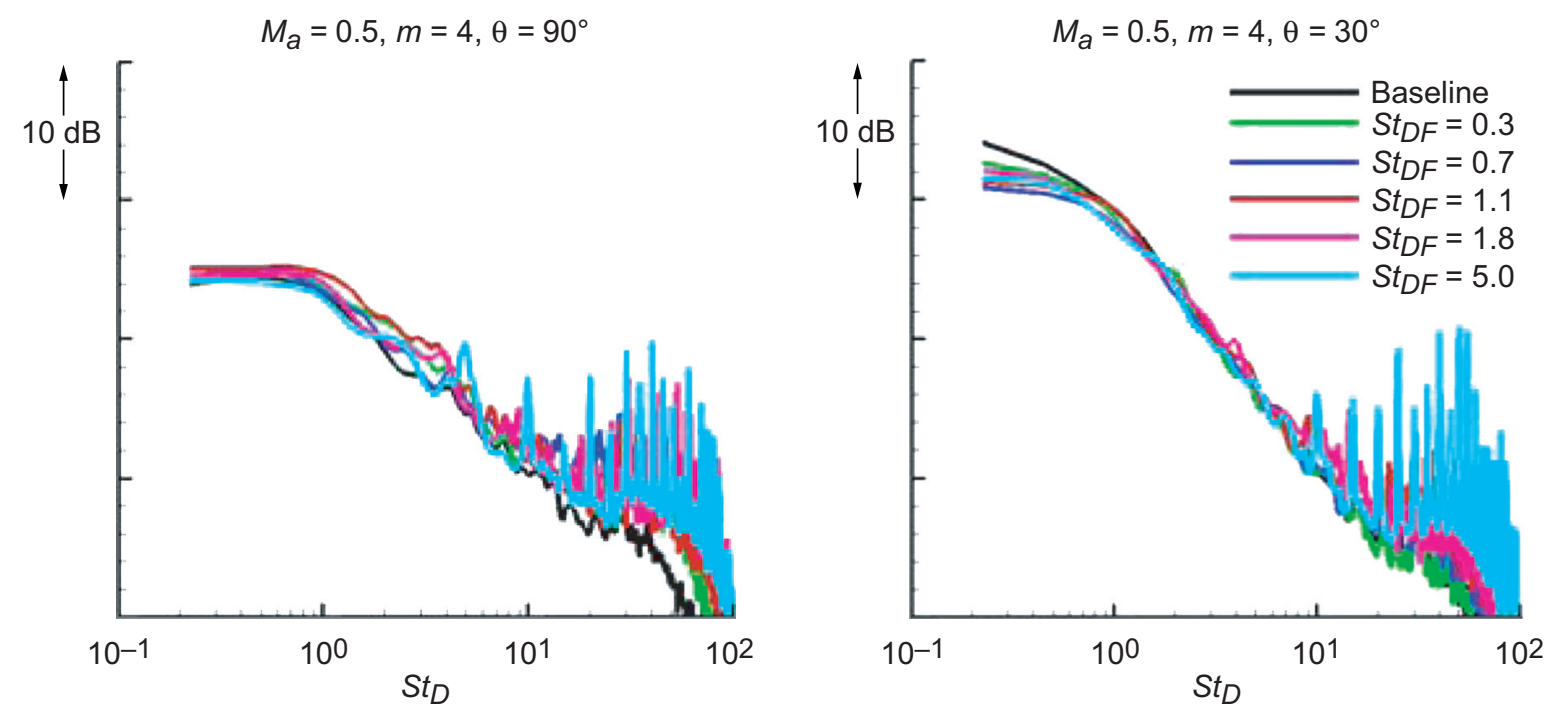

Figure 18.-Far field noise spectra for $M_{a}=0.5$ jet at NASA for forcing at various Strouhal number with azimuthal mode $m=+4$.
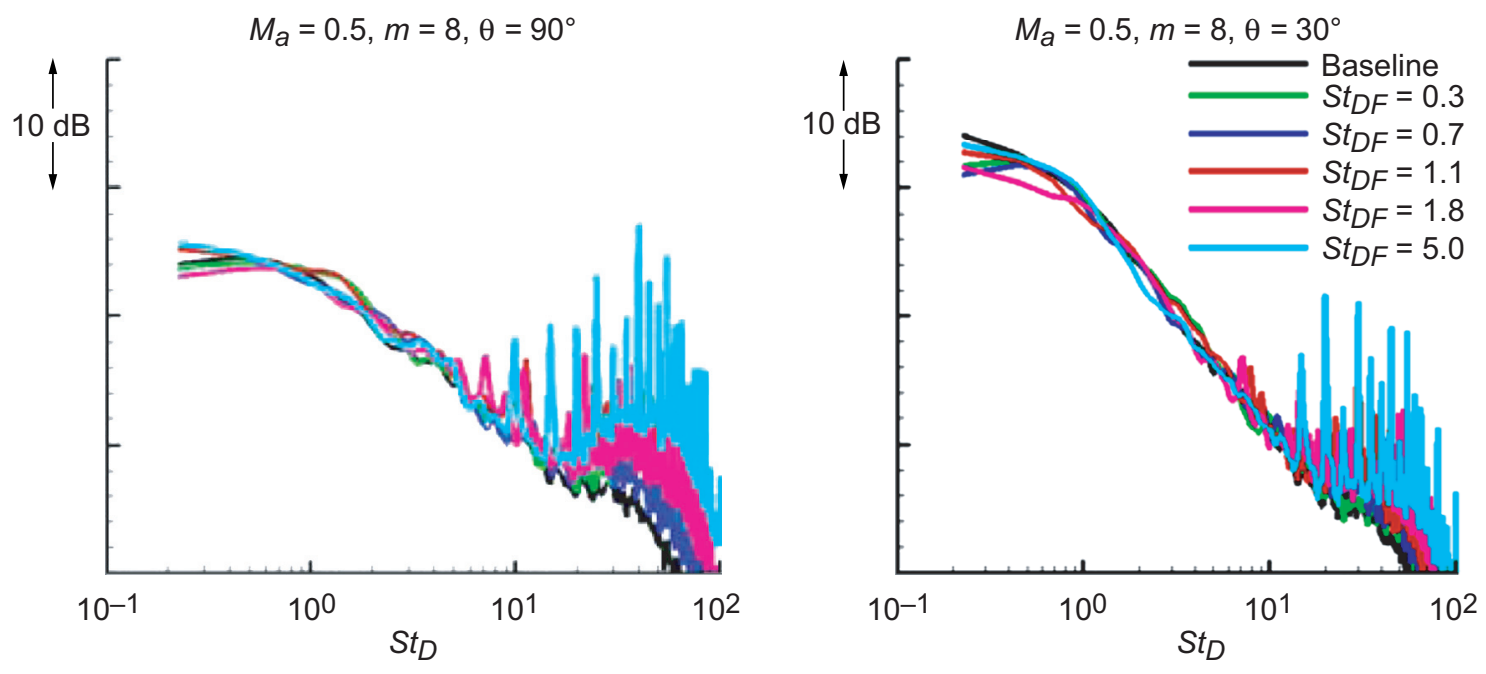

Figure 19.-Far field noise spectra for $M_{a}=0.5$ jet at NASA for forcing at various Strouhal number with azimuthal mode $m=+8$.

Figure 18 and 19 shows spectra for forcing at NASA the $m=4$ and $m=8$, respectively. For $m=4$ and at $30^{\circ}$, the noise is reduced with all forcing frequencies. At $90^{\circ}$ the broadband level is similar to the baseline jet. For $m=8$, the suppression of the broadband at $30^{\circ}$ is similar to that seen when forcing $m=4$.

The ability to force higher azimuthal modes appears to be advantageous when looking for noise mitigation. Many authors have reported on the first few azimuthal modes containing most of the far-field energy with the higher modes being less efficient radiators. The results shown at both facilities seem to support this conclusion. The higher Mach number jet was also forced with azimuthal modes, $m, 4$ and 8 at NASA (not shown here), but similar to the $m= \pm 1$ results (see fig. 16), the lack of sufficient actuators seemed to inhibit the actuator authority. 


\section{Concluding Remarks}

The idea of manipulating flow to change its characteristics is over a century old. Manipulating instabilities of a jet to increase its mixing and to reduce its radiated noise started in 1970s. While the effort has been successful in low-speed and low Reynolds number jets, available actuators' capabilities in terms of their amplitude, bandwidth, and phasing have fallen short in control of high-speed and high Reynolds number jets of practical interest. Over the past couple of years, a class of high amplitude and high bandwidth actuators called localized arc filament plasma actuators (LAFPA) have been developed at OSU and extensively used at GDTL for control of high-speed and high Reynolds number jets. The results of the work has been presented and published in the literature. While the technique has been quite successful and is very promising, all the work up to this point had been carried out using small high subsonic and low supersonic jets from a $2.54 \mathrm{~cm}$ diameter nozzle exit with a Reynolds number of about a million.

The preliminary work reported in this paper is a first attempt to evaluate the scalability of the technique. The power supply/plasma generator was designed and built in-house at GDTL to operate 8 actuators simultaneously over a large frequency range $(0$ to $200 \mathrm{kHz})$, covering over the jet instabilities frequencies, with independent control over phase and duty cycle of each actuator. This allowed forcing the small jet at GDTL with azimuthal modes $m=0,1,2,3, \pm 1, \pm 2$, and \pm 4 over a wide range of frequencies. This power supply was literally taken to and used at NASA with relatively minor modifications. At NASA, a total of 32 actuators were distributed around the 7.5 in. nozzle at NATR. A linear increase with nozzle exit diameter would require 60 actuators at NATR. LAFPA design does not require any change with nozzle scale. However, it is expected that the number of actuators will linearly increase with nozzle exit diameter. With this arrangement at NATR only 8 actuators could operate simultaneously, thus limiting the forcing of the jet at NASA to only three azimuthal modes $m= \pm 1,4$, and 8.

Very preliminary results at NASA indicate that the trends observed in the larger NASA facility in terms of the effects of actuation frequency and azimuthal modes are similar to the smaller GDTL facility. This was most prominent at the lower Mach number $\left(M_{a}=0.5\right)$. However, the actuation authority seems to fall short in the larger jet at higher Mach numbers, resulting in decreased amplitude response compared to the small jet, which is attributed at this point to the lack of sufficient number of actuators. The preliminary results seem also to suggest that amplitude of actuation tones is similar in both the small and larger jets.

\section{References}

Ahuja, K.K., Lepicovsky, J., and Burrin, R.H., "Noise and Flow Structure of a Tone-Excited Jet," AIAA Journal, Vol. 20, No. 12, 1982, pp. 1700-1706.

Ahuja, K.K. and Blakney, D.F., "Tone Excited Jets, Acoustic Measurements," Journal of Sound and Vibration, Vol. 102, 1985, pp. 93-117.

Bridges, J. and Wernet, M.P., "Cross-Stream PIV Measurements of Jets with Internal Lobed Mixers," AIAA Paper 2004-2896 (2004).

Brown, G.L. and Roshko, A., "On Density Effects and Large Structure in Turbulent Mixing Layers," Journal of Fluid Mechanics, Vol. 64, 1974, pp. 715-816.

Crow, S. and Champagne, F., "Orderly Structure in Jet Turbulence," Journal of Fluid Mechanics, Vol. 48, 1971, pp. 547-591.

Ho, C.-M. and Huerre, P., "Perturbed Free Shear Layers," Annual Review of Fluid Mechanics, Vol. 16, 1984, pp. 365-424.

Jubelin, B., "New Experimental Studies on Jet Noise Amplification," AIAA Paper 80-0961, 1980.

Kastner, J., Hileman, J., and Samimy, M., "Exploring High-Speed Axisymmetric Jet Noise Control Using Hartmann Tube Fluidic Actuators," AIAA Paper 2004-0186, 2004.

Kastner, J., Kim, J.-H., and Samimy, M., "Noise Sources in Controlled High Reynolds Number and High Speed Jets," AIAA Paper 2006-2650, 2006. 
Kibens, V. "Discrete Noise Spectrum Generated by an Acoustically Excited Jet," AIAA Journal, Vol. 18, 1980, pp. 434-451.

Lighthill, M.J., "On Sound Generated Aerodynamically I. General Theory," Proc. of the Royal Society of London, Vol. 211, 1952, pp. 564-586.

Lighthill, M.J., "On Sound Generated Aerodynamically II. Turbulence as a Sound Source," Proc. of the Royal Society of London, Vol. 222, 1954, pp. 1-32.

Lu, H.Y., "Effect of Excitation on Coaxial Jet Noise," AIAA Journal, Vol. 21, 1983, pp. 214-220.

Michalke, A. and Fuchs, H.V., "On turbulence and noise of an axisymmetric shear flow," Journal of Fluid Mechanics, Vol. 70, 1975, pp. 179-205.

Moore, C.J., "The role of shear-layer instability waves in jet exhaust noise," Journal of Fluid Mechanics, Vol. 80, 1977, pp. 321-367.

Samimy, M., Adamovich, I., Webb, B., Kastner, J., Hileman, J., Keshav, S., and Palm, P., "Development and Characterization of Plasma Actuators for High Speed Jet Control," Experiments in Fluids, Vol. 37, No. 4, 2004, pp. 577-588.

Samimy, M., Kim, J.-H., Adamovich, I., Utkin, Y., and Keshav, S., "High Speed Jet Control Using Plasma Actuators," 4th International Symposium on Turbulence and Shear Flow Phenomena, Williamsburg, Virginia, June 2005.

Samimy, M., Kim, J.-H., Adamovich, I., Utkin, Y., and Kastner, J., "Active Control of High Speed and High Reynolds Number Free Jet Using Plasma Actuators," 44th AIAA Aerospace Sciences Meeting and Exhibit, Invited paper, AIAA Paper 2006-0711, 2006a.

Samimy, M., Kim, J.-H., Adamovich, I., Utkin, Y., and Kastner, J., "Toward Noise Mitigation in High Speed and High Reynolds Number Jets Using Plasma Actuators," AIAA Paper 2006-2703, 2006b.

Zaman, K.B.M.Q. and Hussain, A.K.M.F., "Turbulence suppression in free shear flows by controlled excitation," Journal of Fluid Mechanics, Vol. 103, 1981, pp. 133-159. 


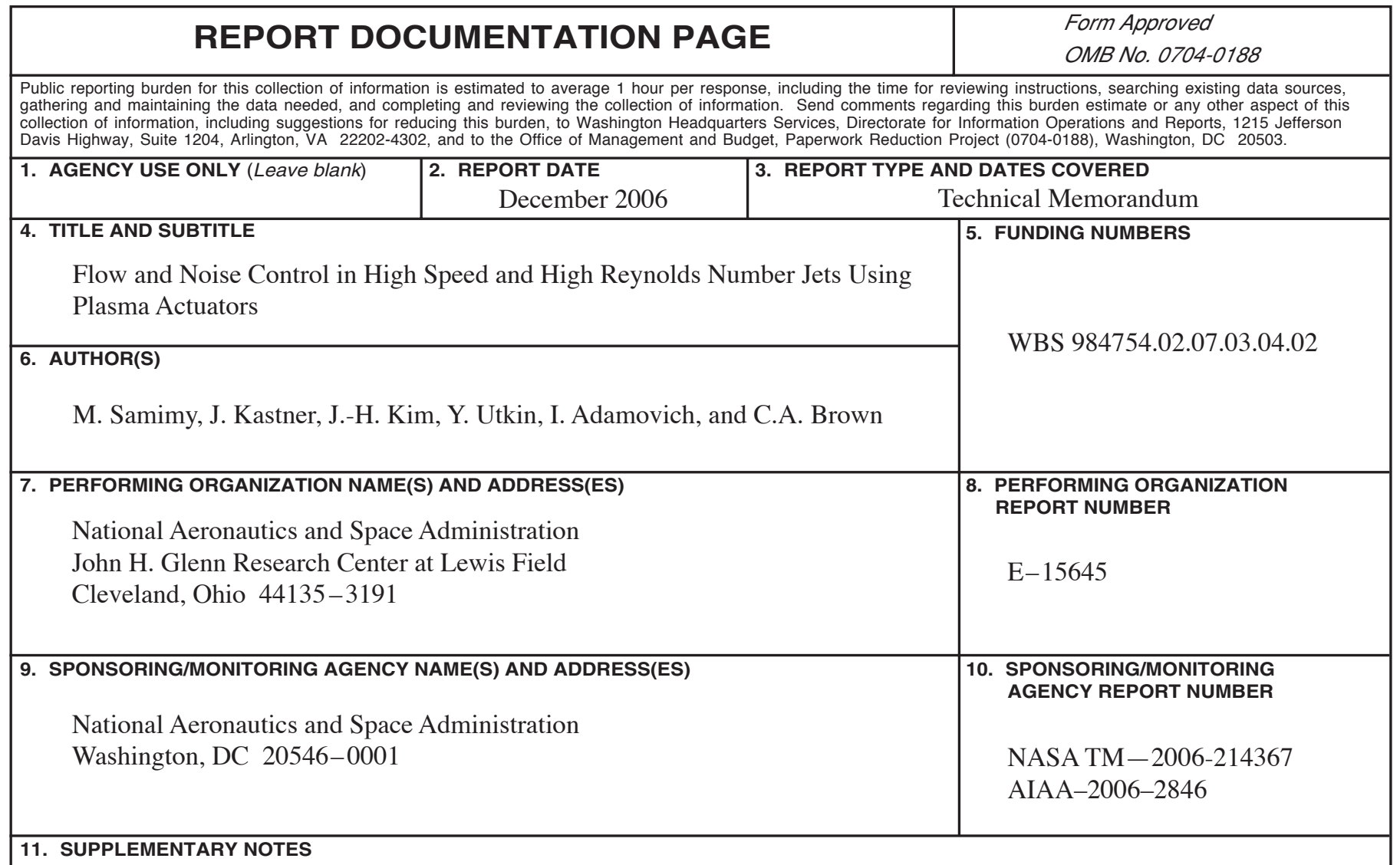

Prepared for the Third Flow Control Conference sponsored by the American Institute of Aeronautics and Astronautics, San Francisco, California, June 5-8, 2006. Responsible person, Clifford A. Brown, organization code RTA, 216-433-2896.

\begin{tabular}{|l|l|l|l|l}
\hline 12a. DISTRIBUTION/AVAILABILITY STATEMENT & 12b. DISTRIBUTION CODE
\end{tabular}

Unclassified - Unlimited

Subject Categories: 07, 09, and 34

Available electronically at http://gltrs.grc.nasa.gov

This publication is available from the NASA Center for AeroSpace Information, 301-621-0390.

13. ABSTRACT (Maximum 200 words)

Localized arc filament plasma actuators have recently been developed and extensively used at Gas Dynamics and

Turbulence Laboratory (GDTL) for control of high-speed and high Reynolds number jets. While the technique has been quite successful, all the work up to this point had been carried out using small high subsonic and low supersonic jets from a $2.54 \mathrm{~cm}$ diameter nozzle exit with a Reynolds number of about a million. The preliminary work reported in this paper is a first attempt to evaluate the scalability of the technique. The localized arc filament plasma actuators were installed and tested at the NASA Nozzle Acoustic Test Rig (NATR) using a 7.5 in. diameter nozzle. Very preliminary results at NATR indicate that the trends observed in the larger NASA facility in terms of the effects of actuation frequency and azimuthal modes are similar in both small GDTL and larger NASA jets. However, the actuation authority seems to fall short in the larger jet at higher Mach numbers, which is attributed at this point to the lack of sufficient number of actuators.

14. SUBJECT TERMS

Jet flow; Aeroacoustics; Shear layer; Actuator; Plasma (physics); Kelvin-Helmholtz instability; Active control

\begin{tabular}{|c|c|c|}
\hline $\begin{array}{c}\text { 17. SECURITY CLASSIFICATION } \\
\text { OF REPORT } \\
\text { Unclassified }\end{array}$ & $\begin{array}{c}\text { 18. SECURITY CLASSIFICATION } \\
\text { OF THIS PAGE } \\
\text { Unclassified }\end{array}$ & $\begin{array}{c}\text { 19. SECURITY CLASSIFICATION } \\
\text { OF ABSTRACT } \\
\text { Unclassified }\end{array}$ \\
\hline
\end{tabular}

NSN 7540-01-280-5500

Standard Form 298 (Rev. 2-89)

Prescribed by ANSI Std. Z39-18 298-102 

